\title{
NONTANGENCY-BASED LYAPUNOV TESTS FOR CONVERGENCE AND STABILITY IN SYSTEMS HAVING A CONTINUUM OF EQUILIBRIA*
}

\author{
SANJAY P. BHAT ${ }^{\dagger}$ AND DENNIS S. BERNSTEIN ${ }^{\ddagger}$
}

\begin{abstract}
This paper focuses on the stability analysis of systems having a continuum of equilibria. Two notions that are of particular relevance to such systems are convergence and semistability. Convergence is the property whereby every solution converges to a limit point that may depend on the initial condition. Semistability is the additional requirement that all solutions converge to limit points that are Lyapunov stable. We give new Lyapunov-function-based results for convergence and semistability of nonlinear systems. These results do not make assumptions of sign definiteness on the Lyapunov function. Instead, our results use a novel condition based on nontangency between the vector field and invariant or negatively invariant subsets of the level or sublevel sets of the Lyapunov function or its derivative and represent extensions of previously known stability results involving semidefinite Lyapunov functions. To illustrate our results we deduce convergence and semistability of the kinetics of the Michaelis-Menten chemical reaction and the closed-loop dynamics of a scalar system under a universal adaptive stabilizing feedback controller.
\end{abstract}

Key words. nontangency, Lyapunov stability, semistability, convergence, prolongations

AMS subject classifications. 34C99, 34D20, 37B25, 93D99

DOI. $10.1137 / \mathrm{S} 0363012902407119$

1. Introduction. This paper focuses on the stability analysis of systems that have a continuum of equilibria. Examples of such systems include mechanical systems having rigid-body modes and isospectral matrix dynamical systems [15]. Such systems also arise in chemical kinetics, compartmental modeling, and adaptive control. Since every neighborhood of a nonisolated equilibrium contains another equilibrium, a nonisolated equilibrium cannot be asymptotically stable. Thus asymptotic stability is not the appropriate notion of stability for systems having a continuum of equilibria. However, given a system that has a continuum of equilibria, it is still natural to ask if the trajectories converge to limit points and if the limit points are Lyapunov stable. These questions lead us to consider the properties of convergence and semistability. For linear systems, semistability was originally defined in [9] and applied to matrix second-order systems in [3]. In the present paper, we extend the notion of semistability to nonlinear systems. Preliminary versions of some of the results of this paper appeared in $[5,6]$.

Convergence is the notion that every trajectory of the system converges to a limit point. The limit point, which is necessarily an equilibrium, depends in general on the initial conditions. In a convergent system, the limit points of trajectories may or may not be Lyapunov stable. Semistability is the additional requirement that trajectories converge to limit points that are Lyapunov stable. More precisely, an equilibrium is semistable if it is Lyapunov stable, and every trajectory starting in a neighborhood

*Received by the editors May 3, 2002; accepted for publication (in revised form) April 23, 2003; published electronically, November 14, 2003. A preliminary version of this paper appeared in the Proceedings of the American Control Conference, Arlington, VA, 2001.

http://www.siam.org/journals/sicon/42-5/40711.html

${ }^{\dagger}$ Department of Aerospace Engineering, Indian Institute of Technology, Powai, Mumbai 400076, India (bhat@aero.iitb.ac.in).

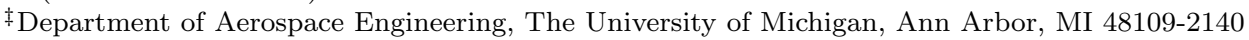
(dsbaero@engin.umich.edu). This author was supported in part by the Air Force Office of Scientific Research under grant F49620-98-1-0037. 
of the equilibrium converges to a (possibly different) Lyapunov stable equilibrium. It can be seen that, for an equilibrium, asymptotic stability implies semistability, while semistability implies Lyapunov stability.

The relationship between Lyapunov stability, semistability, and asymptotic stability can be understood by considering the motion of a particle translating along a fixed direction. Such a particle, when moving under the action of a linear elastic spring, possesses a unique equilibrium, which is Lyapunov stable. In the additional presence of viscous damping, all motions of the particle converge to the unique equilibrium state, which is thus asymptotically stable. On the other hand, a particle moving under the action of viscous damping in the absence of a position-dependent restoring force can remain at rest in any position and thus exhibits a continuum of equilibria, each of which is Lyapunov stable. All motions of such a particle converge to rest, and the equilibrium that the particle converges to is determined by the initial position and velocity of the particle. The motion of the particle is thus convergent, while every equilibrium of the dynamics is semistable.

Besides the damped motion of a particle, there are several applications which involve systems having a continuum of equilibria, and in which semistability is the appropriate notion of stability. For example, we can consider the stability of the lateral dynamics of an aircraft in level trimmed flight. For disturbances affecting the angle between the longitudinal axis and the velocity vector, the vertical tail is designed to influence yaw so as to cause the sideslip angle to converge to zero. However, the heading angle will not generally converge to its predisturbance value. The offset in the final heading angle is an indication of the existence of a continuum of semistable equilibria.

Another application of semistability involves the kinetics of chemical reactions. While periodic or chaotic behavior can occur in chemical reactions [27], it is of interest to determine conditions under which the concentrations of the reacting species converge. In this case, the limiting concentrations are not completely determined by the dynamics but depend upon the initial concentrations as well. The stability of chemical kinetics with respect to a stoichiometric subspace is considered in [11, 12, 28], while [4] applies Lyapunov theory to study the semistability of mass action chemical kinetics.

Chemical reactions are a special case of a more general class of systems known as compartmental systems, which involve mass or energy balance [18]. Compartmental systems arise in biomedical, environmental, economic, power, and thermodynamic applications. Since compartmental systems possess a continuum of equilibria, semistability is the appropriate notion of stability.

In control applications, it is often desirable to design the control system so that the closed-loop system, in the absence of exogenous inputs (commands and disturbances), has an equilibrium that is asymptotically stable. For such designs, semistability is not needed. However, adaptive controllers [17, 22, 23, 24] involve feedback gains that evolve in response to the plant trajectories; that is, the limiting values of the gains depend on the initial condition of the plant states. An adaptive closed-loop system is thus not asymptotically stable, yet convergence and Lyapunov stability of the plant/gain equilibria, that is, semistability, is desirable.

In all of the applications above, it is of interest to determine the convergence and semistability properties of the system. Accordingly, we wish to obtain Lyapunov tests for convergence and semistability.

It is obvious that if a system is convergent, then all of its trajectories converge 
to the set of equilibria. However, as the following example shows, the converse is not true.

Example 1.1. Consider the system $\dot{y}(t)=f(y(t))$, where $f: \mathbb{R}^{2} \rightarrow \mathbb{R}^{2}$ is the continuous vector field given by

$$
\begin{aligned}
f(x)= & \operatorname{sign}\left(x_{1}^{2}+x_{2}^{2}-1\right)\left|x_{1}^{2}+x_{2}^{2}-1\right|^{\alpha} f_{r}(x) \\
& +\operatorname{sign}\left(x_{1}^{2}+x_{2}^{2}-1\right)\left|x_{1}^{2}+x_{2}^{2}-1\right|^{\beta} f_{\theta}(x),
\end{aligned}
$$

with $\alpha, \beta \geq 1$ and the vector fields $f_{r}$ and $f_{\theta}$ given by

$$
f_{r}(x)=\left[\begin{array}{l}
-x_{1} \\
-x_{2}
\end{array}\right], f_{\theta}(x)=\left[\begin{array}{c}
x_{2} \\
-x_{1}
\end{array}\right] \text {. }
$$

The vector fields $f_{r}$ and $f_{\theta}$ point in the radial and circumferential directions, respectively, and thus the parameters $\alpha$ and $\beta$ determine the rates at which solutions move in these directions, respectively. This can be seen more clearly by rewriting (1) in terms of polar coordinates $r=\sqrt{x_{1}^{2}+x_{2}^{2}}$ and $\theta=\tan ^{-1}\left(x_{2} / x_{1}\right)$ as

$$
\begin{aligned}
\dot{r} & =-r \operatorname{sign}\left(r^{2}-1\right)\left|r^{2}-1\right|^{\alpha}, \\
\dot{\theta} & =-\operatorname{sign}\left(r^{2}-1\right)\left|r^{2}-1\right|^{\beta} .
\end{aligned}
$$

It can be seen from (3) and (4) that the set of equilibria $f^{-1}(0)$ consists of the origin $x=0$ and the unit circle $\mathrm{S}^{1}=\left\{x \in \mathbb{R}^{2}: x_{1}^{2}+x_{2}^{2}=1\right\}$. All solutions of the system starting from nonzero initial conditions $y(0)$ that are not on the unit circle approach the unit circle. Solutions starting outside the unit circle spiral in clockwise toward the unit circle, while solutions starting inside the unit circle spiral out counterclockwise. Consequently, all solutions are bounded, and, for every choice of $\alpha$ and $\beta$, all solutions converge to the set of equilibria. However, if $\alpha \geq \beta+1$, then the system is not convergent. This can be seen by using (3) and (4) to obtain

$$
\frac{d r}{d \theta}=r\left|r^{2}-1\right|^{\alpha-\beta}
$$

For initial values $r(0)>1$, solutions of (5) converge to the equilibrium value $r=1$ for decreasing $\theta$, while, for initial values $r(0)<1$, solutions converge to $r=1$ for increasing $\theta$. For $\alpha \geq \beta+1$, the right-hand side of (5) is locally Lipschitz in $r$. Consequently, (5) has a unique maximally defined solution given by $r \equiv 1$ for the initial condition $r(0)=1$ for increasing as well as decreasing $\theta$. Since convergence to the equilibrium value $r=1$ on a finite $\theta$-interval for increasing (decreasing) $\theta$ implies nonuniqueness of solutions for decreasing (increasing) $\theta$ for the initial condition $r(0)=1$, it follows that the solution $r(\cdot)$ of (5) can approach $r=1$ only as $\theta \rightarrow \infty$ if $r(0)<1$ and as $\theta \rightarrow-\infty$ if $r(0)>1$. This implies that, for $\alpha-\beta \geq 1$, the solutions of (3)-(4) that converge to the unit circle spiral around an infinite number of times, thus ruling out convergence. In subsequent sections, we will use the results of this paper to show convergence and semistability in the case $\alpha \leq \beta$. Figure 1 shows the phase portrait of the system for $\alpha=2$ and $\beta=1$, while Figure 2 shows the phase portrait of the system for $\alpha=\beta=1$.

In the case of Figure 1, where $\alpha=2$ and $\beta=1$, it follows from (5) that $\frac{d r}{d \theta} \rightarrow 0$ as $r \rightarrow 1$ so that all nontrivial trajectories approach the unit circle tangentially. As shown above, the system depicted in Figure 1 is not convergent, and, moreover, every equilibrium on the unit circle is unstable. In contrast, all trajectories in Figure 2, 


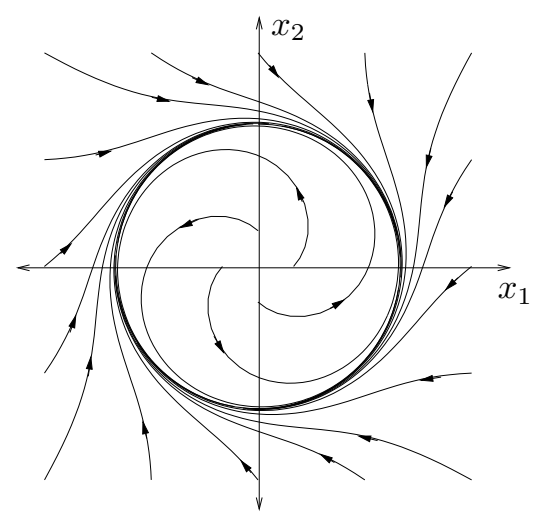

FIG. 1. Phase portrait of (3)-(4) for $\alpha=2, \beta=1$.

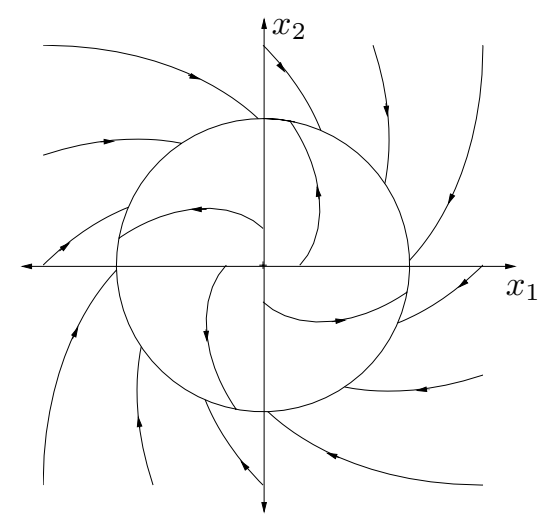

FIG. 2. Phase portrait of (3)-(4) for $\alpha=\beta=1$.

where $\alpha=\beta=1$, approach the unit circle nontangentially, the system is convergent, and all equilibria on the unit circle are semistable. Thus, Figures 1 and 2 suggest that nontangency of trajectories to the set of equilibria is a sufficient condition under which convergence to the set of equilibria implies convergence and semistability.

Intuitively, a vector field is nontangent to a set at a point if the vector field at the point is not contained in the tangent space to the set at that point. We shall apply this intuitive idea to the situation depicted in Figure 2, where the vector field is the vector field describing the dynamics and the set is the set of equilibria of the system. However, this intuitive notion presents two chief difficulties when the set under consideration is the set of singular points of the vector field, that is, the set of equilibria of the system. First, the vector field at an equilibrium is zero, and hence it is always contained in the tangent space to the set of equilibria. To capture the notion of nontangency in such a case, we introduce the direction cone of a vector field in section 4. The second difficulty is that, unlike as in Figures 1 and 2, the set of equilibria may not be sufficiently regular to possess a tangent space at the equilibrium point under consideration and may have corners or self-intersections. For example, consider a dynamical system that evolves on the nonnegative orthant and has the boundary of the orthant as its set of equilibria. In this case, the set of equilibria has a corner at the origin. We overcome this difficulty by considering the tangent 
cone $[2,26]$, which extends the notion of a tangent space to a nonsmooth setting. In section 4 , we formalize our intuitive notion of nontangency by defining nontangency of a vector field to a set at a point to be the condition that the tangent cone to the set at the point and the direction cone of the vector field at that point have no nonzero vector in common. Section 4 contains examples illustrating direction cones as well as nontangency. We also present a result that is useful in computing the direction cone of a vector field in applications.

We apply our notion of nontangency in section 5 , where we show that the solution starting from a point converges if and only if the vector field is nontangent to the positive limit set of the point at some positive limit point. While this result cannot be applied directly in practice, we use it along with well-known properties of positive limit sets to show that, if the vector field is nontangent to the largest invariant subset of the zero-level set of the derivative of a Lyapunov function that is nonincreasing along the solutions, then every bounded solution converges to a limit.

Since the application of the convergence results of section 5 depend on verifying the boundedness of trajectories, we consider the property of boundedness in section 3 . We extend well-known results for boundedness involving proper (that is, radially unbounded in the case where the state-space is $\mathbb{R}^{n}$ ) Lyapunov functions $[21,30]$ by introducing the notion of a weakly proper function. A function is weakly proper if the connected components of its sublevel sets are compact. We show that the existence of a weakly proper function that is nonincreasing along the trajectories implies that the trajectories are bounded. The usefulness of this result is illustrated in the examples given in section 3 .

In section 6 , we apply nontangency to Lyapunov stability. Here, prolongations $[7,8]$ play a role analogous to that played by positive limit sets in section 5 . More specifically, we introduce the restricted prolongation of a point and show that an equilibrium point is Lyapunov stable if and only if the vector field is nontangent at the equilibrium to its restricted prolongation. The restricted prolongation of a point is a subset of its positive prolongation as defined in $[7,8]$. While positive prolongations have been widely used in stability analysis [7], restricted prolongations have invariance properties that are needed for the results that we present. These properties, which are established in section 6, represent one of the key contributions of this paper.

In section 7, we use the results of sections 5 and 6 to obtain novel Lyapunov tests for Lyapunov stability, semistability, and asymptotic stability of nonlinear systems. These results do not make any assumptions about the sign definiteness of the Lyapunov function. Instead, they require only that the Lyapunov function derivative be nonpositive and the equilibrium be a local minimizer of the Lyapunov function on the set of points at which the Lyapunov function derivative is negative. For Lyapunov stability, the weaker assumptions on the Lyapunov function are supplemented by assuming nontangency of the vector field to invariant or negatively invariant subsets of the level set of the Lyapunov function containing the equilibrium and, for semistability, to invariant or negatively invariant subsets of the zero-level set of the Lyapunov function derivative. These results either extend or complement known results for Lyapunov stability and asymptotic stability involving semidefinite Lyapunov functions and Lyapunov function derivatives as given in [1, 16, 20, 21].

As mentioned above, chemical kinetics comprise one of the application areas for semistability theory. Since the kinetic equation for a system of chemical reactions governs concentrations of the reacting species, all solutions of physical interest take values in the nonnegative orthant. For such systems, which evolve on possibly closed 
positively invariant subsets of $\mathbb{R}^{n}$, it is natural to consider relative stability, that is, stability with respect to perturbed initial conditions that belong to the positively invariant subset. Therefore, with applications to nonnegative dynamics in mind, we consider relative stability of dynamical systems that evolve on (not necessarily open) subsets of $\mathbb{R}^{n}$. Relative stability has been considered previously in $[7,16]$.

We illustrate the main results by applying them to examples from chemical kinetics and adaptive control. More specifically, we use the nontangency-based Lyapunov results to deduce convergence and semistability of the kinetics of the MichaelisMenten chemical reaction [11] and the closed-loop dynamics of a scalar system under a universal adaptive stabilizing controller given in [17, 22].

2. Preliminaries. Let $\mathcal{G} \subseteq \mathbb{R}^{n}$, and let $\|\cdot\|$ denote a norm on $\mathbb{R}^{n}$. A subset $\mathcal{U}$ of $\mathcal{G}$ is relatively open in $\mathcal{G}$ if $\mathcal{U}$ is open in the subspace topology induced on $\mathcal{G}$ by the norm $\|\cdot\|$. Given $\mathcal{K} \subseteq \mathcal{G}$, we let int $\mathcal{K}$ and bd $\mathcal{K}$ denote the interior and boundary, respectively, of $\mathcal{K}$ in the subspace topology on $\mathcal{G}$. Thus int $\mathcal{K}$ is the largest subset of $\mathcal{K}$ that is relatively open in $\mathcal{G}$, while bd $\mathcal{K}=(\overline{\mathcal{K}} \cap \mathcal{G}) \backslash$ int $\mathcal{K}$, where $\overline{\mathcal{K}}$ denotes the closure of $\mathcal{K}$ in $\mathbb{R}^{n}$. A set $\mathcal{U} \subseteq \mathcal{G}$ is relatively bounded in $\mathcal{G}$ if $\overline{\mathcal{U}}$ is compact and contained in $\mathcal{G}$. A point $x \in \mathbb{R}^{n}$ is a subsequential limit of a sequence $\left\{x_{i}\right\}$ in $\mathbb{R}^{n}$ if there exists a subsequence of $\left\{x_{i}\right\}$ that converges to $x$ in the norm $\|\cdot\|$. A sequence $\left\{x_{i}\right\}$ in $\mathcal{G}$ is relatively bounded in $\mathcal{G}$ if it is relatively bounded when viewed as a set. Every sequence that is relatively bounded in $\mathcal{G}$ has at least one subsequential limit, and every subsequential limit of the sequence is contained in $\mathcal{G}$. When there is no possibility of confusion, we will use "relatively open (bounded)" instead of "relatively open (bounded) in $\mathcal{G}$." Also, in the case where $\mathcal{G}=\mathbb{R}^{n}$, we will use "open" and "bounded" instead of "relatively open" and "relatively bounded," respectively.

We recall that a set $\mathcal{K} \subseteq \mathcal{G}$ is connected if and only if every pair of relatively open sets $\mathcal{U}_{i} \subseteq \mathcal{G}, i=1,2$, satisfying $\mathcal{K} \subseteq \mathcal{U}_{1} \cup \mathcal{U}_{2}$ and $\mathcal{U}_{i} \cap \mathcal{K} \neq \varnothing, i=1,2$, has a nonempty intersection. Also, a connected component of the set $\mathcal{K} \subseteq \mathcal{G}$ is a connected subset of $\mathcal{K}$ that is not properly contained in any connected subset of $\mathcal{K}$.

Consider the system of differential equations

$$
\dot{y}(t)=f(y(t)),
$$

where $f: \mathcal{D} \rightarrow \mathbb{R}^{n}$ is continuous on the open set $\mathcal{D} \subseteq \mathbb{R}^{n}$. We assume that, for every initial condition $y(0) \in \mathcal{D}$ and every $a>0$, the differential equation (6) possesses a unique $\mathrm{C}^{1}$ solution $y:[0, a) \rightarrow \mathcal{D}$ on the interval $[0, a)$. Letting $\psi(\cdot, x)$ denote the solution of $(6)$ that exists on $[0, \infty)$ and satisfies the initial condition $y(0)=x$, the above assumptions imply that the map $\psi:[0, \infty) \times \mathcal{D} \rightarrow \mathcal{D}$ is continuous [14, Thm. V.2.1], satisfies $\psi(0, x)=x$, and possesses the semigroup property, that is, $\psi(t, \psi(h, x))=\psi(t+h, x)$ for all $t, h \geq 0$ and $x \in \mathcal{D}$. Given $t \geq 0$, it will often be convenient to denote the map $\psi(t, \cdot): \mathcal{D} \rightarrow \mathcal{D}$ by $\psi_{t}$. The orbit $\mathcal{O}_{x}$ of a point $x \in \mathcal{D}$ is the set $\{\psi(t, x): t \geq 0\}$.

A set $\mathcal{U} \subseteq \mathbb{R}^{n}$ is positively invariant if $\psi_{t}(\mathcal{U}) \subseteq \mathcal{U}$ for all $t \geq 0$. The set $\mathcal{U}$ is negatively invariant if, for every $z \in \mathcal{U}$ and every $t \geq 0$, there exists $x \in \mathcal{U}$ such that $\psi(t, x)=z$ and $\psi(\tau, x) \in \mathcal{U}$ for all $\tau \in[0, t]$. Hence, if $\mathcal{U}$ is negatively invariant, then $\mathcal{U} \subseteq \psi_{t}(\mathcal{U})$ for all $t \geq 0$, although the converse is not generally true. Finally, the set $\mathcal{U}$ is invariant if $\psi_{t}(\mathcal{U})=\mathcal{U}$ for all $t \geq 0$. Note that a set is invariant if and only if it is positively as well as negatively invariant. Also, it is easy to show that each connected component of a positively invariant (respectively, negatively invariant, invariant) set is positively invariant (respectively, negatively invariant, invariant). 
In the rest of the paper, $\mathcal{G} \subseteq \mathcal{D}$ will denote a positively invariant set so that $\mathcal{O}_{x} \subseteq \mathcal{G}$ for all $x \in \mathcal{G}$. Except for local compactness, which is invoked in section 4 and subsequent sections, we require no additional hypotheses on $\mathcal{G}$.

An equilibrium point of (6) is a point $x \in \mathcal{D}$ satisfying $f(x)=0$ or, equivalently, $\psi(t, x)=x$ for all $t \geq 0$. We let $\mathcal{E}=f^{-1}(0) \cap \mathcal{G}$, the set of all equilibrium points of $(6)$ in $\mathcal{G}$. An isolated equilibrium is an isolated point of $\mathcal{E}$. An equilibrium point $x \in \mathcal{E}$ is Lyapunov stable relative to $\mathcal{G}$ if, for every relatively open neighborhood $\mathcal{U}_{\varepsilon} \subseteq \mathcal{G}$ of $x$, there exists a relatively open neighborhood $\mathcal{U}_{\delta} \subseteq \mathcal{G}$ of $x$ such that $\psi_{t}\left(\mathcal{U}_{\delta}\right) \subseteq \mathcal{U}_{\varepsilon}$ for all $t \geq 0$. If $x \in \mathcal{E}$ is Lyapunov stable relative to $\mathcal{G}$, then every relatively open neighborhood of $x$ contains a positively invariant, relatively open neighborhood of $x$ [8, section V.1].

The system (6) is convergent relative to $\mathcal{G}$ if, for every $x \in \mathcal{G}, \lim _{t \rightarrow \infty} \psi(t, x)$ exists and is contained in $\mathcal{G}$. It follows from the continuity of $\psi$ and the semigroup property that, if $x \in \mathcal{G}$ is such that $\lim _{t \rightarrow \infty} \psi(t, x)$ exists and is contained in $\mathcal{G}$, then, for every $h>0, \psi_{h}\left(\lim _{t \rightarrow \infty} \psi(t, x)\right)=\lim _{t \rightarrow \infty} \psi(t+h, x)=\lim _{t \rightarrow \infty} \psi(t, x)$ so that $\lim _{t \rightarrow \infty} \psi(t, x) \in \mathcal{E}$.

An equilibrium point $x \in \mathcal{G}$ is semistable relative to $\mathcal{G}$ if there exists a relatively open neighborhood $\mathcal{U} \subseteq \mathcal{G}$ of $x$ such that, for every $z \in \mathcal{U}$, $\lim _{t \rightarrow \infty} \psi(t, z)$ exists, is contained in $\mathcal{G}$, and is Lyapunov stable relative to $\mathcal{G}$. Note that if the equilibrium $x \in \mathcal{G}$ is semistable relative to $\mathcal{G}$, then every equilibrium in some relatively open neighborhood of $x$ is Lyapunov stable relative to $\mathcal{G}$. In particular, every equilibrium that is semistable relative to $\mathcal{G}$ is also Lyapunov stable relative to $\mathcal{G}$.

An equilibrium point $x \in \mathcal{G}$ is asymptotically stable relative to $\mathcal{G}$ if $x$ is Lyapunov stable relative to $\mathcal{G}$ and there exists a relatively open neighborhood $\mathcal{U} \subseteq \mathcal{G}$ of $x$ such that, for every $z \in \mathcal{U}, \lim _{t \rightarrow \infty} \psi(t, z)=x$. It is easy to see that an equilibrium is asymptotically stable relative to $\mathcal{G}$ if and only if it is an isolated equilibrium and is semistable relative to $\mathcal{G}$.

Given a function $V: \mathcal{G} \rightarrow \mathbb{R}$, a point $x \in \mathcal{G}$ is a local minimizer of $V$ relative to $\mathcal{K} \subseteq \mathcal{G}$ if there exists a relatively open neighborhood $\mathcal{U} \subseteq \mathcal{G}$ of $x$ such that $V(x) \leq V(z)$ for all $z \in \mathcal{U} \cap \mathcal{K}$. The point $x \in \mathcal{G}$ is a global minimizer of $V$ relative to $\mathcal{G}$ if $V(x) \leq V(z)$ for all $z \in \mathcal{G}$. Local and global maximizers of $V$ are defined similarly.

Given a continuous function $V: \mathcal{G} \rightarrow \mathbb{R}$, we define

$$
\dot{V}(x)=\lim _{h \rightarrow 0^{+}} \frac{1}{h}[V(\psi(h, x))-V(x)]
$$

for every $x \in \mathbb{R}^{n}$ such that the limit in (7) exists. It is easy to see that if $x \in \mathcal{E}$ and $V: \mathcal{G} \rightarrow \mathbb{R}$, then $\dot{V}(x)$ is defined and equals zero.

Some of the results that we present involve an equilibrium point that is also a local or global maximizer of $\dot{V}$ for some function $V$. Since $\dot{V}$ is zero at equilibrium points, an equilibrium $x$ is a local maximizer of $\dot{V}$ relative to $\mathcal{G}$ if and only if $\dot{V}$ assumes nonpositive values in some relatively open neighborhood of $x$. Similarly, an equilibrium $x$ is a global maximizer of $\dot{V}$ relative to $\mathcal{G}$ if and only if $\dot{V}$ assumes nonpositive values in $\mathcal{G}$. We will find it convenient to state the familiar requirements of local and global negative semidefiniteness on $\dot{V}$ in terms of the equilibrium being a local or global maximizer, respectively, of $\dot{V}$.

3. Boundedness of orbits. A function $U: \mathcal{G} \rightarrow \mathbb{R}$ is proper if $U^{-1}(\mathcal{I})$ is a compact subset of $\mathcal{G}$ for all compact subsets $\mathcal{I}$ of $\mathbb{R}$, and weakly proper if, for every $c \in \mathbb{R}$, every connected component of the set $\{x \in \mathcal{G}: U(x) \leq c\}=U^{-1}((-\infty, c])$ is compact. If $U$ is proper and bounded below on $\mathcal{G}$, then $U$ is weakly proper. 
We present a Lyapunov test for boundedness of orbits that will be useful in some of the examples we present in this paper. The test involves weakly proper functions.

Proposition 3.1. Suppose there exists a weakly proper, continuous function $U: \mathcal{G} \rightarrow \mathbb{R}$ such that $\dot{U}$ is defined on $\mathcal{G}$ and such that $\dot{U}(x) \leq 0$ for all $x \in \mathcal{G}$. Then, for every $x \in \mathcal{G}, \mathcal{O}_{x}$ is relatively bounded in $\mathcal{G}$.

Proof. Consider $x \in \mathcal{G}$, and let $c=U(x)$. The assumptions on $\dot{U}$ imply that the function $t \mapsto U(\psi(t, x))$ is nonincreasing. Hence $U(\psi(t, x)) \leq c$ for all $t \geq 0$ so that $\psi(t, x) \in \mathcal{K} \stackrel{\text { def }}{=}\{z \in \mathcal{G}: U(z) \leq c\}$ for all $t \geq 0$. Thus $\mathcal{O}_{x} \subseteq \mathcal{K}$. Since $\mathcal{O}_{x}$ is connected, it follows that $\mathcal{O}_{x}$ is contained in a connected component $\mathcal{M}$ of $\mathcal{K}$. By weak properness, $\mathcal{M}$ is compact, and thus $\overline{\mathcal{O}}_{x}$ is contained in $\mathcal{M}$. Hence $\mathcal{O}_{x}$ is relatively bounded in $\mathcal{G}$.

Proposition 3.1 is an extension of the following well-known sufficient condition for boundedness of orbits. See, for instance, [21, Thm. 4], [30, Thm. 8.7].

Corollary 3.1. Suppose there exists a proper, continuous function $U: \mathcal{G} \rightarrow \mathbb{R}$ such that $\dot{U}$ is defined on $\mathcal{G}$ and such that $U(x) \geq 0$ and $\dot{U}(x) \leq 0$ for all $x \in \mathcal{G}$. Then, for every $x \in \mathcal{G}, \mathcal{O}_{x}$ is relatively bounded in $\mathcal{G}$.

Proof. The result follows from Proposition 3.1 by noting that the function $U$ is weakly proper.

Example 3.1. Consider the uncertain linear system

$$
\dot{y}(t)=-a y(t)+b u(t),
$$

where $a, b \in \mathbb{R}$ and $b$ is nonzero but otherwise unknown. A universal adaptive stabilizing controller for the system (8) is given by [17, 22, 24]

$$
u(t)=-k^{2}(t) \cos k(t) y(t),
$$

where the adaptive gain parameter $k$ satisfies the update law

$$
\dot{k}(t)=y^{2}(t) .
$$

The corresponding closed-loop system on $\mathcal{G}=\mathbb{R}^{2}$ is described by (10) and

$$
\dot{y}(t)=-\left[a+b k^{2}(t) \cos (k(t))\right] y(t) .
$$

We will use Proposition 3.1 to show that all orbits of the closed-loop system (10)-(11) are bounded.

Consider the function $U: \mathbb{R}^{2} \rightarrow \mathbb{R}$ given by $U(x)=\frac{1}{2} y^{2}+g(k)$, where $x=(y, k)$ and $g: \mathbb{R} \rightarrow \mathbb{R}$ is given by $g(k)=a k+b\left(k^{2}-2\right) \sin k+2 b k \cos k$. It is easy to verify that, for the closed-loop system, $\dot{U}(x)=0$ for every $x \in \mathbb{R}^{2}$. We claim that the function $U$ is weakly proper.

Let $c \in \mathbb{R}$, let $\mathcal{K}=\left\{x \in \mathbb{R}^{2}: U(x) \leq c\right\}$, and consider $x_{0}=\left(y_{0}, k_{0}\right) \in \mathcal{K}$. Let $\mathcal{M}$ be the connected component of $\mathcal{K}$ containing $x_{0}$. By continuity of $U, \mathcal{K}$ is closed. Since $\mathcal{M}$ is a connected component of a closed set, $\mathcal{M}$ is closed.

First suppose $b>0$, and let $p: \mathbb{R} \rightarrow \mathbb{R}$ be given by $p(k)=b k^{2}+a k-2 b$. There exists an integer $m>0$ such that, for $k_{1}=-\left(2 m+\frac{3}{2}\right) \pi$ and $k_{2}=\left(2 m+\frac{1}{2}\right) \pi$, it follows that $p\left(k_{i}\right)>c, i=1,2$, and $k_{0} \in\left[k_{1}, k_{2}\right]$. A simple computation shows that $p\left(k_{i}\right)=g\left(k_{i}\right), i=1,2$, so that, for every $y \in \mathbb{R}$, the points $x_{i}=\left(y, k_{i}\right), i=1,2$, satisfy $U\left(x_{i}\right)=\frac{1}{2} y^{2}+p\left(k_{i}\right)>c$. Letting $\rho$ denote the projection $(y, k) \mapsto k$, it follows that $\rho(\mathcal{M})$ is a connected set that contains $k_{0} \in\left[k_{1}, k_{2}\right]$ but does not contain $k_{1}$ and $k_{2}$. Hence it follows that $\rho(\mathcal{M}) \subseteq\left[k_{1}, k_{2}\right]$; that is, $k \in\left[k_{1}, k_{2}\right]$ for every 
$x=(y, k) \in \mathcal{M}$. Denote $l=\min _{k \in\left[k_{1}, k_{2}\right]} g(k)$. Then, for every $x=(y, k) \in \mathcal{M}$, $\frac{1}{2} y^{2} \leq c-g(k) \leq c-l$. Thus, for every $x=(y, k) \in \mathcal{M}, y \in\left[y_{1}, y_{2}\right]$, where $y_{1}=-\sqrt{2(c-l)}$ and $y_{2}=\sqrt{2(c-l)}$. Thus the closed set $\mathcal{M}$ is contained in the compact set $\left[y_{1}, y_{2}\right] \times\left[k_{1}, k_{2}\right]$ and hence compact. It follows that $U$ is weakly proper in the case where $b>0$.

Now suppose $b<0$, and let $p: \mathbb{R} \rightarrow \mathbb{R}$ be given by $p(k)=-b k^{2}+a k+2 b$. There exists an integer $m>0$ such that, for $k_{1}=-\left(2 m+\frac{1}{2}\right) \pi$ and $k_{2}=\left(2 m+\frac{3}{2}\right) \pi$, it follows that $p\left(k_{i}\right)>c, i=1,2$, and $k_{0} \in\left[k_{1}, k_{2}\right]$. Letting $l=\min _{k \in\left[k_{1}, k_{2}\right]} g(k)$, $y_{1}=-\sqrt{2(c-l)}$, and $y_{2}=\sqrt{2(c-l)}$, it can be shown by repeating the arguments given above that the closed set $\mathcal{M}$ is contained in the compact set $\left[y_{1}, y_{2}\right] \times\left[k_{1}, k_{2}\right]$ and hence compact. It follows that $U$ is weakly proper in the case where $b<0$.

Since the function $U$ is weakly proper and $\dot{U} \equiv 0$, it follows from Proposition 3.1 that every orbit of the closed-loop system (10)-(11) is bounded.

4. Direction cones and nontangency. Given a set $\mathcal{K} \subseteq \mathbb{R}^{n}$, we let co $\mathcal{K}$ denote the union of the convex hulls of the connected components of $\mathcal{K}$ and let coco $\mathcal{K}$ denote the cone generated by co $\mathcal{K}$. Given $\mathcal{K} \subseteq \mathbb{R}^{n}$ and $x \in \mathbb{R}^{n}$, we denote $\operatorname{dist}(x, \mathcal{K})=$ $\inf _{y \in \mathcal{K}}\|x-y\|$. Finally, we let $\mathrm{S}^{n-1}=\left\{x \in \mathbb{R}^{n}:\|x\|=1\right\}$ denote the unit sphere in $\mathbb{R}^{n}$.

Given $x \in \mathcal{G}$, the direction cone $\mathcal{F}_{x}$ of $f$ at $x$ relative to $\mathcal{G}$ is the intersection of all sets of the form $\overline{\operatorname{coco}(f(\mathcal{U}) \backslash\{0\})}$, where $\mathcal{U} \subseteq \mathcal{G}$ is a relatively open neighborhood of $x$. It is easy to see that if $x \in \mathcal{G} \backslash$ int $\mathcal{E}$, then $\mathcal{F}_{x}$ is a closed cone containing $f(x)$, while if $x \in$ int $\mathcal{E}$, then $\mathcal{F}_{x}=\varnothing$.

Let $\mathcal{K} \subseteq \mathbb{R}^{n}$ and $x \in \mathcal{K}$. A vector $v \in \mathbb{R}^{n}$ is tangent to $\mathcal{K}$ at $x \in \mathcal{K}$ if there exist a sequence $\left\{x_{i}\right\}$ in $\mathcal{K}$ converging to $x$ and a sequence $\left\{h_{i}\right\}$ of positive real numbers converging to 0 such that $\lim _{i \rightarrow \infty} \frac{1}{h_{i}}\left(x_{i}-x\right)=v$. The tangent cone to $\mathcal{K}$ at $x$ is the closed cone $T_{x} \mathcal{K}$ of all vectors tangent to $\mathcal{K}$ at $x$ [2, p. 121], [26, Prop. 6.2]. It is easy to see that $0 \in T_{x} \mathcal{K}$. Moreover, if $x$ is an isolated point of $\mathcal{K}$, then $T_{x} \mathcal{K}=\{0\}$. Also, if $\mathcal{K} \subseteq \mathcal{M}$, then, for every $x \in \mathcal{K}, T_{x} \mathcal{K} \subseteq T_{x} \mathcal{M}$. Finally, if $x \in \mathcal{K}$ and $\mathcal{U} \subseteq \mathbb{R}^{n}$ is an open neighborhood of $x$ such that $\mathcal{K} \cap \mathcal{U}$ is a differentiable submanifold of $\mathbb{R}^{n}$, then $T_{x} \mathcal{K}$ is the usual tangent space to $\mathcal{K}$ at $x$.

Remark 4.1. Tangent cones are called contingent cones in [2]. We have followed the terminology used in [26].

The vector field $f$ is nontangent to the set $\mathcal{K} \subseteq \mathcal{G}$ at the point $x \in \mathcal{K}$ relative to $\mathcal{G}$ if $T_{x} \mathcal{K} \cap \mathcal{F}_{x} \subseteq\{0\}$.

Remark 4.2. The notion of nontangency introduced here is different from the well-known notion of transversality [13]. Transversality between a vector field and a set is possible only at a point in the set where the vector field is not zero and the set is locally a differentiable submanifold of codimension one. On the other hand, nontangency is possible even if the vector field is zero and the set is not a differentiable submanifold of codimension one.

In the rest of the paper, we assume that $\mathcal{G}$ is locally compact, that is, every point in $\mathcal{G}$ is contained in a relatively open and relatively bounded set $\mathcal{U} \subseteq \mathcal{G}$. In particular, if $\mathcal{G}$ is either open or closed, then $\mathcal{G}$ is locally compact. Local compactness implies that every relatively open neighborhood of a point $x \in \mathcal{G}$ contains a relatively open neighborhood of $x$ that is also relatively bounded in $\mathcal{G}$ [10, Thm. XI.6.2].

The following proposition is a key result of this paper. The result shows that if the vector field $f$ is nontangent to the set $\mathcal{B}$ of all subsequential limits of sequences of points taken from a sequence of segments of orbits of (6), then the set $\mathcal{B}$ contains exactly one point. This result will be applied to positive limit sets in section 5 
and restricted prolongations in section 6 to obtain nontangency-based conditions for convergence and stability.

Proposition 4.1. Let $x \in \mathcal{G}$, and let $\left\{x_{i}\right\}$ be a sequence in $\mathcal{G}$ converging to $x$. Let $\mathcal{I}_{i} \subseteq[0, \infty), i=1,2, \ldots$, be intervals containing 0 , and let $\mathcal{B} \subseteq \mathcal{G}$ be the set of all subsequential limits contained in $\mathcal{G}$ of sequences of the form $\left\{\psi\left(\tau_{i}, x_{i}\right)\right\}$, where $\tau_{i} \in \mathcal{I}_{i}$ for each $i$. Then $\mathcal{B}=\{x\}$ if and only if $f$ is nontangent to $\mathcal{B}$ at $x$ relative to $\mathcal{G}$.

Proof. First, we note that $x \in \mathcal{B}$ since $x=\lim _{i \rightarrow \infty} \psi\left(0, x_{i}\right)$. Necessity now follows by noting that if $\mathcal{B}=\{x\}$, then $T_{x} \mathcal{B}=\{0\}$, and hence $T_{x} \mathcal{B} \cap \mathcal{F}_{x} \subseteq\{0\}$.

To prove sufficiency, suppose $z_{0} \in \mathcal{B}, z_{0} \neq x$. If the sequence $\left\{f\left(x_{i}\right)\right\}$ is eventually zero, then every sequence of the form $\left\{\psi\left(\tau_{i}, x_{i}\right)\right\}$ converges to $x$, and, consequently, $\mathcal{B}$ is a singleton. Hence we may assume without loss of generality that $f\left(x_{i}\right) \neq 0$ for every $i$. Let $\left\{\mathcal{U}_{k}\right\}$ be a nested sequence of neighborhoods of $x$ that are relatively bounded and relatively open in $\mathcal{G}$, contained in $\mathcal{U}$, and such that $\overline{\mathcal{U}_{k+1}} \subset \mathcal{U}_{k}$ and $x_{k} \in \mathcal{U}_{k}$ for every $k=1,2, \ldots, \cap_{k} \mathcal{U}_{k}=\{x\}$, and $z_{0} \notin \mathcal{U}_{1}$. Since $z_{0} \in \mathcal{B}$, there exists a sequence $\left\{\tau_{i}\right\}$ such that $\tau_{i} \in \mathcal{I}_{i}$ for every $i$, and $\lim _{i \rightarrow \infty} \psi\left(\tau_{i}, x_{i}\right)=z_{0} \notin \mathcal{U}_{1}$. By continuity of $\psi$, for every $k$, there exists a sequence $\left\{h_{j}^{k}\right\}_{j=k}^{\infty}$ in $[0, \infty)$ such that, for every $j \geq k$, $h_{j}^{k} \in \mathcal{I}_{j}, h_{j}^{k} \leq \tau_{j}, \psi\left(\tau, x_{j}\right) \in \mathcal{U}_{k}$ for every $\tau \in\left[0, h_{j}^{k}\right)$, and $\psi\left(h_{j}^{k}, x_{j}\right) \in \operatorname{bd} \mathcal{U}_{k}$. For each $k$, let $z_{k} \in \operatorname{bd} \mathcal{U}_{k}$ be a subsequential limit of the relatively bounded sequence $\left\{\psi\left(h_{j}^{k}, x_{j}\right)\right\}_{j=k}^{\infty}$. Then, for every $k$, it follows that $z_{k} \in \mathcal{B}, z_{k} \neq x$, and $\lim _{k \rightarrow \infty} z_{k}=x$. Now consider a subsequential limit $v$ of the bounded sequence $\left\{\left\|z_{k}-x\right\|^{-1}\left(z_{k}-x\right)\right\}$. Clearly, $v \in T_{x} \mathcal{B}$. Also, $\|v\|=1$ so that $v \neq 0$. We claim that $v \in \mathcal{F}_{x}$.

Let $\mathcal{V} \subseteq \mathcal{G}$ be a relatively open neighborhood of $x$, and consider $\varepsilon>0$. By construction, there exists $k$ such that $\|v-\| z_{k}-x\left\|^{-1}\left(z_{k}-x\right)\right\|<\varepsilon / 3$. Moreover, since $\cap_{i} \mathcal{U}_{i}=\{x\}$, we can assume that $\mathcal{U}_{k} \subseteq \mathcal{V}$. Since $z_{k}$ belongs to the boundary of a relatively open neighborhood of $x, \delta \stackrel{\text { def }}{=}\left\|z_{k}-x\right\|>0$. Since $z_{k}=\lim _{i \rightarrow \infty} \psi\left(h_{i}^{k}, x_{i}\right)$ and $x=\lim _{i \rightarrow \infty} x_{i}$, there exists $i$ such that $x_{i} \in \mathcal{V},\left\|x-x_{i}\right\|<\varepsilon \delta / 3$, and $\left\|z_{k}-\psi\left(h_{i}^{k}, x_{i}\right)\right\|<$ $\varepsilon \delta / 3$. Let $\mathcal{A}$ be the connected component of $f(\mathcal{V}) \backslash\{0\}$ containing $f\left(x_{i}\right) \neq 0$. Since $f$ is continuous, it follows that $f\left(\psi\left(\tau, x_{i}\right)\right) \in \overline{\mathcal{A}}$ for all $\tau \in\left[0, h_{i}^{k}\right]$. Therefore, $w \stackrel{\text { def }}{=}$ $\psi\left(h_{i}^{k}, x_{i}\right)-x_{i}=\int_{0}^{h_{i}^{k}} f\left(\psi\left(\tau, x_{i}\right)\right) d \tau$ is contained in the convex cone generated by $\overline{\mathcal{A}}[29$, Thm. I.6.13]. Since $\mathcal{A}$ is connected, coco $\mathcal{A}$ is simply the convex cone generated by $\mathcal{A}$. Since $\mathcal{A}$ and $\overline{\mathcal{A}}$ generate the same closed convex cone, we have coco $\overline{\mathcal{A}} \subseteq \overline{\operatorname{coco}} \overline{\mathcal{A}}=$ $\overline{\operatorname{coco} \mathcal{A}} \subseteq \overline{\operatorname{coco}(f(\mathcal{V}) \backslash\{0\})}$. Thus $w \in \overline{\operatorname{coco}(f(\mathcal{V}) \backslash\{0\})}$. Now,

$$
\begin{aligned}
\left\|v-\delta^{-1} w\right\| & =\left\|v-\delta^{-1}\left(z_{k}-x\right)-\delta^{-1}\left(\psi\left(h_{i}^{k}, x_{i}\right)-z_{k}\right)-\delta^{-1}\left(x-x_{i}\right)\right\| \\
& \leq\|v-\| z_{k}-x\left\|^{-1}\left(z_{k}-x\right)\right\|+\delta^{-1}\left\|\psi\left(h_{i}^{k}, x_{i}\right)-z_{k}\right\|+\delta^{-1}\left\|x-x_{i}\right\| \\
& <\varepsilon .
\end{aligned}
$$

We conclude that, for every $\varepsilon>0$, there exists $w \in \overline{\operatorname{coco}(f(\mathcal{V}) \backslash\{0\})}$ and $\delta>0$ such

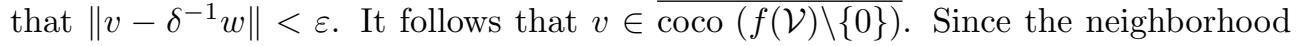
$\mathcal{V}$ was arbitrary, it follows that $v \in \mathcal{F}_{x}$. Thus, if $\mathcal{B} \neq\{x\}$, then there exists $v \in \mathbb{R}^{n}$ such that $v \neq 0$ and $v \in T_{x} \mathcal{B} \cap \mathcal{F}_{x}$; that is, $f$ is not nontangent to $\mathcal{B}$ at $x$ relative to $\mathcal{G}$. Sufficiency now follows.

Since any application of Proposition 4.1 will involve finding the direction cone, we next give a result that provides a convenient means of determining the direction cone in applications. For this purpose, it will be useful to introduce the limiting direction set of a vector field at a point.

Let $x \in \mathcal{G} \backslash$ int $\mathcal{E}$. A vector $v \in \mathrm{S}^{n-1}$ is a limiting direction of $f$ at $x$ relative to $\mathcal{G}$ if there exists a sequence $\left\{x_{i}\right\}$ in $\mathcal{G} \backslash \mathcal{E}$ such that $\lim _{i \rightarrow \infty} x_{i}=x$ and 
$\lim _{i \rightarrow \infty} \frac{1}{\left\|f\left(x_{i}\right)\right\|} f\left(x_{i}\right)=v$. The limiting direction set $\mathcal{L}_{x}$ of $f$ at $x$ relative to $\mathcal{G}$ is the set of all limiting directions of $f$ at $x$ relative to $\mathcal{G}$. Clearly, $\mathcal{L}_{x}$ is nonempty, compact, and contained in $\mathrm{S}^{n-1}$. Moreover, for every $\varepsilon>0$, there exists a relatively open neighborhood $\mathcal{U}_{\varepsilon} \subseteq \mathcal{G}$ of $x$ such that, for every $z \in \mathcal{U}_{\varepsilon} \backslash \mathcal{E}$, $\operatorname{dist}\left(\frac{1}{\|f(z)\|} f(z), \mathcal{L}_{x}\right)<\varepsilon$.

Consider $x \in \mathcal{G} \backslash$ int $\mathcal{E}$, and let $\mathcal{U} \subseteq \mathcal{G}$ be a relatively open neighborhood of $x$. For every sequence $\left\{x_{i}\right\}$ in $\mathcal{G} \backslash \mathcal{E}$ converging to $x \in \mathcal{G}$, the sequence $\left\{\frac{1}{\left\|f\left(x_{i}\right)\right\|} f\left(x_{i}\right)\right\}$ is eventually in the cone generated by $f(\mathcal{U}) \backslash\{0\}$ and hence in coco $(f(\mathcal{U}) \backslash\{0\})$ so that every subsequential limit of the sequence $\left\{\frac{1}{\left\|f\left(x_{i}\right)\right\|} f\left(x_{i}\right)\right\}$ is contained in coco $(f(\mathcal{U}) \backslash\{0\})$. Since $\mathcal{U}$ was chosen arbitrarily, it follows that $\mathcal{L}_{x} \subseteq \mathcal{F}_{x}$. The following result shows that if no connected component of the limiting direction set contains the origin in its convex hull, then the direction cone is contained in the union of the convex cones generated by the connected components of the limiting direction set. This result provides a convenient means for determining the direction cone in applications.

Proposition 4.2. Let $x \in \mathcal{G} \backslash \operatorname{int} \mathcal{E}$, and suppose $0 \notin \operatorname{co} \mathcal{L}_{x}$. Then $\mathcal{F}_{x} \subseteq \operatorname{coco} \mathcal{L}_{x}$.

Proof. See the appendix.

Remark 4.3. A special case where Proposition 4.2 applies is the case where $\mathcal{L}_{x} \subseteq \mathrm{S}^{n-1}$ is finite. Suppose $x \in \mathcal{G}$ is such that $\mathcal{L}_{x}$ is finite. Then co $\mathcal{L}_{x}=\mathcal{L}_{x} \subseteq \mathrm{S}^{n-1}$, and thus $0 \notin \operatorname{co} \mathcal{L}_{x}$. Moreover, coco $\mathcal{L}_{x}$ is the union of rays generated by the points of $\mathcal{L}_{x}$. Proposition 4.2 implies that $\mathcal{F}_{x} \subseteq \operatorname{coco} \mathcal{L}_{x}$. However, since $\mathcal{L}_{x} \subset \mathcal{F}_{x}$ and since $\mathcal{F}_{x}$ is a cone, the rays generated by points of $\mathcal{L}_{x}$ are contained in $\mathcal{F}_{x}$, that is, coco $\mathcal{L}_{x} \subseteq \mathcal{F}_{x}$. Thus, in the case where the limiting direction set is finite, the direction cone is the union of rays generated by points of the limiting direction set.

The following example shows that, in general, $\mathcal{F}_{x} \nsubseteq \subseteq \operatorname{coco} \mathcal{L}_{x}$.

Example 4.1. Consider the system (6), where $f: \mathbb{R}^{3} \rightarrow \mathbb{R}^{3}$ is given by

$$
f(x)=-\left(x_{1}^{2}+x_{2}^{2}\right)^{6}\left[\begin{array}{c}
x_{1} \\
x_{2} \\
0
\end{array}\right]+\left(x_{1}^{2}+x_{2}^{2}\right)\left[\begin{array}{c}
x_{2} \\
-x_{1} \\
0
\end{array}\right]+\left(x_{1}^{2}+x_{2}^{2}\right)^{2}\left[\begin{array}{l}
0 \\
0 \\
1
\end{array}\right] .
$$

Letting $\mathcal{G}=\mathbb{R}^{3}$, the set of equilibria is $\mathcal{E}=\left\{x \in \mathcal{G}: x_{1}=x_{2}=0\right\}$.

Let $a \in \mathbb{R}$, and consider $x=\left[\begin{array}{lll}0 & 0 & a\end{array}\right]^{\mathrm{T}} \in \mathcal{E}$. To compute $\mathcal{L}_{x}$, it will be convenient to introduce the function $r: \mathcal{G} \rightarrow \mathbb{R}$ given by $r(z)=\sqrt{z_{1}^{2}+z_{2}^{2}}$ and the function $\theta: \mathcal{G} \backslash \mathcal{E} \rightarrow[0,2 \pi)$ such that, for every $z \in \mathcal{G} \backslash \mathcal{E}, z_{1}=r(z) \cos (\theta(z))$ and $z_{2}=r(z) \sin (\theta(z))$. $\mathcal{G} \backslash \mathcal{E}$,

For every $z \in \mathcal{G},\|f(z)\|=(r(z))^{3} \sqrt{1+(r(z))^{2}+(r(z))^{20}}$, while, for every $z \in$ (13) $\frac{1}{\|f(z)\|} f(z)=\frac{1}{\sqrt{1+(r(z))^{2}+(r(z))^{20}}}\left[\begin{array}{c}-(r(z))^{10} \cos (\theta(z))+\sin (\theta(z)) \\ -(r(z))^{10} \sin (\theta(z))-\cos (\theta(z)) \\ r(z)\end{array}\right]$,

where $\|\cdot\|$ denotes the Euclidean norm on $\mathbb{R}^{3}$. Consider a sequence $\left\{x_{i}\right\}$ in $\mathcal{G} \backslash \mathcal{E}$ converging to $x$. Since $\lim _{i \rightarrow \infty} r\left(x_{i}\right)=0$, it is easy to see from (13) that every subsequential limit of the sequence $\left\{\left\|f\left(x_{i}\right)\right\|^{-1} f\left(x_{i}\right)\right\}$ is of the form $[\sin \alpha-\cos \alpha 0]^{\mathrm{T}}$, where $\alpha \in \mathbb{R}$. On the other hand, for every $\alpha \in \mathbb{R}$, the sequence $\left\{x_{i}\right\}$ given by $x_{i}=$ $\left[\frac{1}{i} \cos \alpha \frac{1}{i} \sin \alpha a\right]^{\mathrm{T}}$ converges to $x$ while $\lim _{i \rightarrow \infty}\left\|f\left(x_{i}\right)\right\|^{-1} f\left(x_{i}\right)=[\sin \alpha-\cos \alpha 0]^{\mathrm{T}}$. Hence we conclude that the limiting direction set of $f$ at $x$ is a circle and is given by $\mathcal{L}_{x}=\left\{[\sin \alpha-\cos \alpha 0]^{\mathrm{T}}: \alpha \in \mathbb{R}\right\} . \mathcal{L}_{x}$ is connected, and hence it easily follows that $\operatorname{co} \mathcal{L}_{x}=\left\{w \in \mathbb{R}^{3}: w_{1}^{2}+w_{2}^{2} \leq 0, w_{3}=0\right\}$ and coco $\mathcal{L}_{x}=\left\{w \in \mathbb{R}^{3}: w_{3}=0\right\}$. Note that $0 \in \operatorname{co} \mathcal{L}_{x}$. 


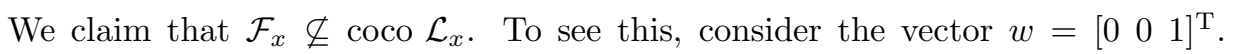
Clearly, $w \notin \operatorname{coco} \mathcal{L}_{x}$. We claim that $w \in \mathcal{F}_{x}$.

Let $\mathcal{U}$ be an open neighborhood of $x$. Choose $\varepsilon>0$ such that $\{z \in \mathcal{G}:\|z-x\| \leq$ $\varepsilon\} \subseteq \mathcal{U}$, and consider $z_{1}=\left[\begin{array}{lll}\varepsilon & 0 & a\end{array}\right]^{\mathrm{T}}$ and $z_{2}=\left[\begin{array}{lll}-\varepsilon & 0 & a\end{array}\right]^{\mathrm{T}}$. Then $z_{i} \in \mathcal{U}, i=1,2$. It is easy to verify that $w=\frac{1}{2} \varepsilon^{-4}\left[f\left(z_{1}\right)+f\left(z_{2}\right)\right]$. Since $\mathcal{U} \backslash \mathcal{E}$ is connected and $f$ is continuous, $f(\mathcal{U}) \backslash\{0\}=f(\mathcal{U} \backslash \mathcal{E})$ is also connected. Therefore, $f\left(z_{i}\right), i=1,2$, are contained in the same connected component of $f(\mathcal{U}) \backslash\{0\}$. Hence $w \in \operatorname{coco}(f(\mathcal{U}) \backslash\{0\})$. Since $\mathcal{U}$ was chosen to be arbitrary, it follows that $w \in \mathcal{F}_{x}$.

The following example illustrates direction cones, nontangency, and the use of Proposition 4.2.

Example 4.2. Consider the system described in Example 1.1 with $\mathcal{G}=\mathbb{R}^{2}$, and let $x \in \mathrm{S}^{1}$. As discussed in Example 1.1, $x$ is an equilibrium point for the system. It is easy to show that

$$
\begin{aligned}
\mathcal{L}_{x} & =\left\{ \pm f_{\theta}(x)\right\}, & & \alpha>\beta, \\
& =\left\{ \pm \frac{1}{\sqrt{2}}\left(f_{\theta}(x)+f_{r}(x)\right)\right\}, & & \alpha=\beta, \\
& =\left\{ \pm f_{r}(x)\right\}, & & \alpha<\beta,
\end{aligned}
$$

where $f_{r}$ and $f_{\theta}$ are given in (2). Thus $\mathcal{L}_{x}$ is finite, and hence, by Remark 4.3,

$$
\begin{aligned}
\mathcal{F}_{x} & =\left\{k f_{\theta}(x): k \in \mathbb{R}\right\}, & & \alpha>\beta, \\
& =\left\{k\left(f_{\theta}(x)+f_{r}(x)\right): k \in \mathbb{R}\right\}, & & \alpha=\beta, \\
& =\left\{k f_{r}(x): k \in \mathbb{R}\right\}, & & \alpha<\beta .
\end{aligned}
$$

The unit circle $\mathrm{S}^{1}$ is a differentiable submanifold of $\mathbb{R}^{2}$. Hence, for every $x \in \mathrm{S}^{1}$, $T_{x} \mathrm{~S}^{1}$ is the tangent line to $\mathrm{S}^{1}$ at $x$. Since the vector field $f_{\theta}$ points in the circumferential direction at every point, it follows that, for every $x \in \mathrm{S}^{1}, T_{x} \mathrm{~S}^{1}=\operatorname{span}\left\{f_{\theta}(x)\right\}$. It now follows from (14) that

$$
\begin{aligned}
T_{x} \mathrm{~S}^{1} \cap \mathcal{F}_{x} & =T_{x} \mathrm{~S}^{1}, \quad \alpha>\beta, \\
& =\{0\}, \quad \alpha \leq \beta .
\end{aligned}
$$

Thus, for every $x \in \mathrm{S}^{1}, f$ is nontangent to $\mathrm{S}^{1}$ at $x$ relative to $\mathcal{G}$ if and only if $\alpha \leq \beta$. It can be observed that Figures 1 and 2 reflect this fact.

5. Positive limit sets, convergence, and nontangency. In this section, we present nontangency-based Lyapunov results for convergence. These results use three key ideas. The first of these, given in Proposition 5.1, is that a solution of (6) converges to a limit if and only if its positive limit set is a singleton set. The second key idea, presented as Proposition 5.2, is to use Proposition 4.1 to show that the positive limit set of a solution of (6) is a singleton set if and only if the vector field $f$ is nontangent to the positive limit set at some point. Since it is not generally possible to find the positive limit set of a solution in practice, it is difficult to check nontangency of the vector field $f$ to the positive limit set in applications. Since nontangency to any outer estimate of the positive limit set implies nontangency to the positive limit set itself, the third key idea is to check nontangency of the vector field $f$ to an outer estimate of the positive limit set that is easier to find in practice. Proposition 5.3 gives outer estimates of the positive limit sets in terms of invariant subsets of the level and sublevel sets of a Lyapunov function and its derivative. Theorem 5.1, the main result of this section, combines the ideas of Propositions 5.1, 5.2, and 5.3 to give 
a sufficient condition for convergence that involves a nontangency condition between the vector field $f$ and invariant subsets of the level sets of the derivative of a Lyapunov function.

Given $x \in \mathcal{G}$, the positive limit set of $x$ relative to $\mathcal{G}$ is the set $\mathcal{O}_{x}^{\infty}$ of points $z \in \mathcal{G}$ such that there exists a divergent sequence $\left\{t_{i}\right\}$ in $[0, \infty)$ satisfying $\lim _{i \rightarrow \infty} \psi\left(t_{i}, x\right)=$ $z$. The first part of the following result on positive limit sets is well known in the case $\mathcal{G}=\mathbb{R}^{n}$. See, for instance, [7, Thm. 5.5, 5.9], [8, p. 24], [19, p. 114], and [21]. The second part depends on the local compactness of $\mathcal{G}$.

Proposition 5.1. Let $x \in \mathcal{G}$. If $\mathcal{O}_{x}$ is relatively bounded in $\mathcal{G}$, then $\mathcal{O}_{x}^{\infty}$ is nonempty, compact, invariant, and connected, and, in addition, $\psi(t, x) \rightarrow \mathcal{O}_{x}^{\infty}$ as $t \rightarrow \infty$; that is, for every relatively open subset $\mathcal{U} \subseteq \mathcal{G}$ that contains $\mathcal{O}_{x}^{\infty}$, there exists $T>0$ such that $\psi(t, x) \in \mathcal{U}$ for all $t>T$. Moreover, $\lim _{t \rightarrow \infty} \psi(t, x)$ exists and is contained in $\mathcal{G}$ if and only if $\mathcal{O}_{x}^{\infty}$ contains exactly one point.

Proof. The first part of the result is well known for $\mathcal{G}=\mathbb{R}^{n}$. The proof is similar in the case where $\mathcal{G} \neq \mathbb{R}^{n}$ and is left to the reader. In the second part, the necessity is straightforward. To prove sufficiency, suppose that $\mathcal{O}_{x}^{\infty}=\{z\}$. Let $\mathcal{U}_{\varepsilon} \subseteq \mathcal{G}$ be a relatively open neighborhood of $z$. Since $\mathcal{G}$ is locally compact, there exists a neighborhood $\mathcal{U} \subseteq \mathcal{U}_{\varepsilon}$ of $x$ that is relatively open and relatively bounded in $\mathcal{G}$. Since $z \in \mathcal{O}_{x}^{\infty}$, there exists a divergent sequence $\left\{t_{i}\right\}$ in $[0, \infty)$ such that $\psi\left(t_{i}, x\right) \in \mathcal{U}$ for all $i$. We claim that there exists $T>0$ such that $\psi(t, x) \in \mathcal{U}_{\varepsilon}$ for all $t>T$. If not, then by the continuity of $\psi$, for every $i$, there exists $\tau_{i}>t_{i}$ such that $\psi\left(\tau_{i}, x\right) \in \operatorname{bd} \mathcal{U}$. In this case, the sequence $\left\{\psi\left(\tau_{i}, x\right)\right\}$ is relatively bounded in $\mathcal{G}$ and hence has a subsequential limit $w \in \mathcal{G}$. By construction, $w \in \operatorname{bd} \mathcal{U}$ and hence $w \neq z$. However, by definition, $w \in \mathcal{O}_{x}^{\infty}=\{z\}$, which is a contradiction. Hence we conclude that there exists $T>0$ such that $\psi(t, x) \in \mathcal{U}_{\varepsilon}$ for all $t>T$. Since $\mathcal{U}_{\varepsilon}$ was chosen arbitrarily, it follows that $\lim _{t \rightarrow \infty} \psi(t, x)=z \in \mathcal{G}$.

The following application of Proposition 4.1 gives a nontangency-based necessary and sufficient condition for a solution of (6) to converge to a limit.

Proposition 5.2. Let $x \in \mathcal{G}$, and suppose that $\mathcal{O}_{x}^{\infty}$ is nonempty. Then $\lim _{t \rightarrow \infty} \psi(t$, $x$ ) exists and is contained in $\mathcal{G}$ if and only if there exists $z \in \mathcal{O}_{x}^{\infty}$ such that $f$ is nontangent to $\mathcal{O}_{x}^{\infty}$ at $z$ relative to $\mathcal{G}$.

Proof. Consider $z \in \mathcal{O}_{x}^{\infty}$. There exists a divergent sequence $\left\{t_{i}\right\}$ in $[0, \infty)$ such that $\lim _{i \rightarrow \infty} \psi\left(t_{i}, x\right)=z$. For every $i$, denote $x_{i}=\psi\left(t_{i}, x\right)$ so that $\lim _{i \rightarrow \infty} x_{i}=z$. $\mathcal{O}_{x}^{\infty}$ is the set of subsequential limits of sequences of the form $\left\{\psi\left(h_{i}, x_{i}\right)\right\}$, where $h_{i} \in[0, \infty)$ for every $i$. Letting $\mathcal{I}_{i}=[0, \infty)$ for every $i$ and $\mathcal{B}=\mathcal{O}_{x}^{\infty}$, it follows from Proposition 4.1 that $\mathcal{O}_{x}^{\infty}=\{z\}$ if and only if $f$ is nontangent to $\mathcal{O}_{x}^{\infty}$ at $z$. The result now follows from the second part of Proposition 5.1.

Example 5.1. Consider the system described in Example 1.1, and assume that $\alpha \geq \beta+1$. Let $\mathcal{G}=\mathbb{R}^{2}$, and consider $x \in \mathcal{G} \backslash \mathrm{S}^{1}$. As discussed in Example 1.1, $\lim _{t \rightarrow \infty} \psi(t, x)$ does not exist. Indeed, $\mathcal{O}_{x}^{\infty}=\mathrm{S}^{1}$. As discussed in Example 4.2, $f$ is not nontangent to $\mathcal{O}_{x}^{\infty}=\mathrm{S}^{1}$ at $x$, thus illustrating Proposition 5.2.

It should be noted that in order to apply Proposition 5.2 to a solution of (6), the positive limit set of the solution needs to be known. Since it is not generally possible to find the positive limit set of a solution, Proposition 5.2 is not directly useful in applications. However, Lyapunov functions can sometimes be used to obtain sets that contain the positive limit set of a solution. The following proposition gives two such containment results for positive limit sets. The result is a straightforward extension of [8, Thm. VIII.6.1, c)] and [21, Thm. 1]. Hence the proof is left to the reader. We note only that the proof uses the invariance properties of positive limit 
sets given in Proposition 5.1.

Proposition 5.3. Suppose $V: \mathcal{G} \rightarrow \mathbb{R}$ is a continuous function such that $\dot{V}$ is defined on $\mathcal{G}$, and $\dot{V}(x) \leq 0$ for all $x \in \mathcal{G}$. Let $x \in \mathcal{G}$ be such that $\mathcal{O}_{x}$ is relatively bounded in $\mathcal{G}$. Let $\mathcal{P}$ and $\mathcal{N}$ denote the largest invariant subsets of the sets $\{z \in \mathcal{G}$ : $V(z) \leq V(x)\}$ and $\dot{V}^{-1}(0)$, respectively. Then $\mathcal{O}_{x}^{\infty} \subseteq \mathcal{P} \cap \mathcal{N}$. In addition, if $\dot{V} \equiv 0$, then $\mathcal{O}_{x}^{\infty}$ is contained in the largest invariant subset of $V^{-1}(V(x))$.

In the following example, we illustrate Propositions 5.2 and 5.3 by applying them to the chemical kinetics of the Michaelis-Menten chemical reaction.

Example 5.2. In the Michaelis-Menten chemical reaction, a substrate $\mathrm{S}$ is converted into a product $\mathrm{P}$ through an intermediate complex $\mathrm{C}$ in the presence of an enzyme E. The reaction is depicted as

$$
\mathrm{S}+\mathrm{E} \underset{k_{2}}{\stackrel{k_{1}}{\rightleftharpoons}} \mathrm{C} \stackrel{k_{3}}{\longrightarrow} \mathrm{P}+\mathrm{E},
$$

where $k_{i}>0, i=1,2,3$, are chemical rate constants. In this example, we use Propositions 5.2 and 5.3 to show that the concentrations of species S, P, C, and E in this chemical reaction converge to equilibrium values.

Letting $y_{1}(t), y_{2}(t), y_{3}(t)$, and $y_{4}(t)$ denote the instantaneous nonnegative concentrations of the species $\mathrm{S}, \mathrm{C}, \mathrm{E}$, and $\mathrm{P}$, respectively, the law of mass action kinetics yields [11]

$$
\dot{y}(t)=y_{2}(t) v_{1}+y_{1}(t) y_{3}(t) v_{2},
$$

where $v_{1}=\left[\begin{array}{llll}k_{2} & -\left(k_{2}+k_{3}\right) & k_{2}+k_{3} & k_{3}\end{array}\right]^{\mathrm{T}}$ and $v_{2}=\left[\begin{array}{llll}-k_{1} & k_{1} & -k_{1} & 0\end{array}\right]^{\mathrm{T}}$. Equation (16) is of the form (6), where $f: \mathbb{R}^{4} \rightarrow \mathbb{R}^{4}$ is given by $f(x)=x_{2} v_{1}+x_{1} x_{3} v_{2}$.

The nonnegative orthant $\mathcal{G}=\left\{x \in \mathbb{R}^{4}: x_{i} \geq 0, i=1, \ldots, 4\right\}$ is positively invariant under the dynamics $(16)[4,11]$. Since the vectors $v_{1}$ and $v_{2}$ are linearly independent, it is easy to see that the set of equilibrium concentrations in $\mathcal{G}$ is $\mathcal{E}=\mathcal{E}_{1} \cup \mathcal{E}_{2}$, where $\mathcal{E}_{1}=\left\{x \in \mathcal{G}: x_{1}=0, x_{2}=0, x_{3}>0\right\}$ and $\mathcal{E}_{2}=\left\{x \in \mathcal{G}: x_{1} \geq 0, x_{2}=0, x_{3}=0\right\}$.

We claim that $f$ is nontangent to $\mathcal{E}_{1}$ at every point in $\mathcal{E}_{1}$ relative to $\mathcal{G}$. Indeed, the direction cone $\mathcal{F}_{x}$ of $f$ at every point $x \in \mathcal{G}$ is contained in the span of $v_{1}$ and $v_{2}$, while every vector that is tangent to $\mathcal{E}_{1}$ at some point $x \in \mathcal{E}_{1}$ is contained in the span of the vectors $v_{3}=\left[\begin{array}{llll}0 & 0 & 1 & 0\end{array}\right]^{\mathrm{T}}$ and $v_{4}=\left[\begin{array}{llll}0 & 0 & 0 & 1\end{array}\right]^{\mathrm{T}}$. Since $\left\{v_{i}: i=1,2,3,4\right\}$ is a set of linearly independent vectors, it follows that $\operatorname{span}\left\{v_{1}, v_{2}\right\} \cap \operatorname{span}\left\{v_{3}, v_{4}\right\}=\{0\}$. Thus $T_{x} \mathcal{E}_{1} \cap \mathcal{F}_{x} \subseteq\{0\}$ for every $x \in \mathcal{E}_{1}$.

It is easy to verify that the function $U: \mathcal{G} \rightarrow \mathbb{R}$ given by $U(x)=x_{1}+2 x_{2}+x_{3}+x_{4}$ is proper and satisfies $\dot{U} \equiv 0$. It follows from Corollary 3.1 that every orbit in $\mathcal{G}$ is relatively bounded in $\mathcal{G}$. Hence, by Proposition $5.1, \mathcal{O}_{x}^{\infty}$ is nonempty for every $x \in \mathcal{G}$.

Now consider the function $V: \mathcal{G} \rightarrow \mathbb{R}$ defined by $V(x)=\frac{1}{2} x_{2}^{2}+x_{1}\left(x_{2}+x_{3}\right)-\frac{1}{2} x_{3}^{2}$. Then $\dot{V}(x)=-k_{3} x_{2}\left(x_{2}+x_{3}\right) \leq 0$ for all $x \in \mathcal{G}$. Thus $\dot{V}^{-1}(0)=\left\{x \in \mathcal{G}: x_{2}=0\right\}$. If a solution $y$ of the differential equation (16) satisfies $y_{2} \equiv 0$, then $\dot{y}_{2} \equiv 0$ and hence $y_{1} y_{3} \equiv 0$. It therefore follows that the largest invariant subset of $\dot{V}^{-1}(0)$ is the set $\mathcal{E}$ of equilibrium concentrations. Proposition 5.3 now implies that $\mathcal{O}_{x}^{\infty} \subseteq \mathcal{E}$ for every $x \in \mathcal{G}$.

Next, consider the function $W: \mathcal{G} \rightarrow \mathbb{R}$ defined by $W(x)=x_{2}+x_{3}$. It is easy to verify that $\dot{W} \equiv 0$. Hence, by Proposition 5.3 , for every $x \in \mathcal{G}, \mathcal{O}_{x}^{\infty}$ is contained in the level set $W^{-1}(W(x))$ of $W$ containing $x$. Thus it follows that, for every $x \in \mathcal{G}$, $\mathcal{O}_{x}^{\infty} \subseteq W^{-1}(W(x)) \cap \mathcal{E}$. Since $W^{-1}(0)=\mathcal{E}_{2}$, it follows that $W^{-1}(W(x)) \cap \mathcal{E} \subseteq \mathcal{E}_{1}$ for every $x \in \mathcal{G}$ satisfying $W(x)>0$. Hence we conclude that, for every $x \in \mathcal{G} \backslash \mathcal{E}_{2}$, $\mathcal{O}_{x}^{\infty} \subseteq \mathcal{E}_{1}$ 
Now consider $x \in \mathcal{E}_{2}$. Then $x$ is an equilibrium, and concentrations starting from the initial value $x$ clearly converge to the equilibrium value $x$. Next, consider $x \in \mathcal{G} \backslash \mathcal{E}_{2}$. Then $\mathcal{O}_{x}^{\infty} \subseteq \mathcal{E}_{1}$ and, for every $z \in \mathcal{O}_{x}^{\infty}, T_{z} \mathcal{O}_{x}^{\infty} \cap \mathcal{F}_{z} \subseteq T_{z} \mathcal{E}_{1} \cap \mathcal{F}_{z} \subseteq\{0\}$. Hence Proposition 5.2 implies that concentrations starting from the initial values $x$ converge to equilibrium values. We conclude that the system described by (16) is convergent relative to $\mathcal{G}$; that is, the concentrations of all species in the MichaelisMenten reaction converge to equilibrium values.

The following result gives a sufficient condition for a trajectory of (6) to converge to a limit. Unlike Proposition 5.2, the following result is not based on nontangency.

Proposition 5.4. Let $x \in \mathcal{G}$. If $\mathcal{O}_{x}^{\infty}$ contains an equilibrium $z$ that is Lyapunov stable relative to $\mathcal{G}$, then $z=\lim _{t \rightarrow \infty} \psi(t, x)$; that is, $\mathcal{O}_{x}^{\infty}=\{z\}$.

Proof. Suppose $z \in \mathcal{O}_{x}^{\infty}$ is Lyapunov stable relative to $\mathcal{G}$. Let $\mathcal{U}_{\varepsilon} \subseteq \mathcal{G}$ be a relatively open neighborhood of $z$. By Lyapunov stability, there exists a relatively open neighborhood $\mathcal{U}_{\delta} \subset \mathcal{G}$ of $z$ such that $\psi_{t}\left(\mathcal{U}_{\delta}\right) \subseteq \mathcal{U}_{\varepsilon}$ for every $t \geq 0$. Since $z \in \mathcal{O}_{x}^{\infty}$, there exists $h \geq 0$ such that $\psi(h, x) \in \mathcal{U}_{\delta}$. Therefore, $\psi(t+h, x)=\psi_{t}(\psi(h, x)) \in$ $\psi_{t}\left(\mathcal{U}_{\delta}\right) \subseteq \mathcal{U}_{\varepsilon}$ for every $t>0$. Since $\mathcal{U}_{\varepsilon} \subseteq \mathcal{G}$ was chosen arbitrarily, it follows that $z=$ $\lim _{t \rightarrow \infty} \psi(t, x)$. It immediately follows that $\lim _{i \rightarrow \infty} \psi\left(t_{i}, x\right)=z$ for every divergent sequence $\left\{t_{i}\right\}$ and thus $\mathcal{O}_{x}^{\infty}=\{z\}$.

Our next result applies Propositions 5.2, 5.3, and 5.4 to obtain a sufficient condition for convergence. The result uses Proposition 5.3 to obtain a set containing all positive limit sets and then uses Propositions 5.2 and 5.4 to show convergence.

TheOREM 5.1. Suppose $\mathcal{O}_{x}$ is relatively bounded in $\mathcal{G}$ for all $x \in \mathcal{G}$, and assume that there exists a continuous function $V: \mathcal{G} \rightarrow \mathbb{R}$ such that $\dot{V}$ is defined on $\mathcal{G}$ and satisfies $\dot{V}(x) \leq 0$ for all $x \in \mathcal{G}$. Let $\mathcal{S} \subseteq \mathcal{E}$ denote the set of equilibria that are Lyapunov stable relative to $\mathcal{G}$, and let $\mathcal{N}_{0}$ denote the largest invariant subset of $\dot{V}^{-1}(0)$. For every $k=0,1,2, \ldots$, let $\mathcal{M}_{k} \subseteq \mathcal{N}_{k}$ denote the set of points in $\mathcal{N}_{k}$ where $f$ is not nontangent to $\mathcal{N}_{k}$ relative to $\mathcal{G}$, and let $\mathcal{N}_{k+1} \subseteq \mathcal{M}_{k}$ denote the largest invariant subset of $\mathcal{M}_{k}$. If $\mathcal{M}_{k} \subseteq \mathcal{S}$ for some $k$, then the system (6) is convergent relative to $\mathcal{G}$.

Proof. Consider $x \in \mathcal{G}$. Since $\mathcal{O}_{x}$ is relatively bounded in $\mathcal{G}$, Proposition 5.1 implies that $\mathcal{O}_{x}^{\infty}$ is nonempty and invariant. To prove the result, we first show that if $\mathcal{O}_{x}^{\infty}$ contains more than one element, then $\mathcal{O}_{x}^{\infty} \subseteq \mathcal{M}_{k} \backslash \mathcal{S}$ for every $k$.

Suppose $\mathcal{O}_{x}^{\infty}$ contains more than one element. By Proposition 5.3, it follows that $\mathcal{O}_{x}^{\infty} \subseteq \mathcal{N}_{0}$. Now assume that $\mathcal{O}_{x}^{\infty} \subseteq \mathcal{N}_{k}$ for some $k=0,1, \ldots$. Since $\mathcal{O}_{x}^{\infty}$ contains more than one element, it follows from Proposition 5.2 that, for every $z \in \mathcal{O}_{x}^{\infty}, f$ is not nontangent to $\mathcal{O}_{x}^{\infty}$ at $z$ relative to $\mathcal{G}$, that is, $\{0\} \nsupseteq T_{z} \mathcal{O}_{x}^{\infty} \cap \mathcal{F}_{z} \subseteq T_{z} \mathcal{N}_{k} \cap \mathcal{F}_{z}$. Since $\mathcal{M}_{k}=\left\{z \in \mathcal{N}_{k}: T_{z} \mathcal{N}_{k} \cap \mathcal{F}_{z} \nsubseteq\{0\}\right\}$, it follows that $\mathcal{O}_{x}^{\infty} \subseteq \mathcal{M}_{k}$. Since $\mathcal{O}_{x}^{\infty}$ is invariant, $\mathcal{O}_{x}^{\infty} \subseteq \mathcal{N}_{k+1}$. It follows by induction that $\mathcal{O}_{x}^{\infty} \subseteq \mathcal{N}_{k+1} \subseteq \mathcal{M}_{k}$ for every $k$. Also, it follows from Proposition 5.4 that $\mathcal{O}_{x}^{\infty} \cap \mathcal{S}=\varnothing$. Thus $\mathcal{O}_{x}^{\infty} \subseteq \mathcal{M}_{k} \backslash \mathcal{S}$ for every $k$.

It follows from the above arguments that if $\mathcal{M}_{k} \backslash \mathcal{S}=\varnothing$ for some $k$, then $\mathcal{O}_{x}^{\infty}$ contains only one element, and hence, by Proposition 5.1, $\lim _{t \rightarrow \infty} \psi(t, x)$ exists and is contained in $\mathcal{G}$. The result now follows.

Example 5.3. In this example, we use Theorem 5.1 to show that the closed-loop adaptive system given by (10) and (11) in Example 3.1 is convergent.

It was shown in Example 3.1 that every orbit of the system (10)-(11) is bounded. Consider the function $V: \mathbb{R}^{2} \rightarrow \mathbb{R}$ given by $V(x)=e^{-k}, x=(y, k) \in \mathbb{R}^{2}$. For the closed-loop system (10)-(11), it follows that $\dot{V}(x)=-e^{-k} y^{2} \leq 0$ for all $x=(y, k) \in$ $\mathbb{R}^{2}$. Thus $\dot{V}^{-1}(0)=\left\{(y, k) \in \mathbb{R}^{2}: y=0\right\}=\mathcal{E}$, the set of equilibria, and the largest invariant subset of $\dot{V}^{-1}(0)$ is $\mathcal{N}_{0}=\dot{V}^{-1}(0)=\mathcal{E}$. 
To investigate nontangency, let $f: \mathbb{R}^{2} \rightarrow \mathbb{R}^{2}$ denote the right-hand side of (10)(11), and let $\mathcal{Z}=\left\{(0, k): a+b k^{2} \cos k=0\right\}$. We note that $\mathcal{Z} \subseteq \mathcal{E}$. Since $b \neq 0$, the set $\mathcal{Z}$ is nonempty, and every point of $\mathcal{Z}$ is isolated. For every $x=(y, k) \in \mathcal{N}_{0} \backslash \mathcal{Z}$ and every sequence $\left\{x_{i}\right\}=\left\{\left(y_{i}, k_{i}\right)\right\}$ in $\mathbb{R}^{2} \backslash \mathcal{E}$ converging to $x$ and satisfying $\operatorname{sign}\left(y_{i}\right)=$ $\operatorname{sign}\left(y_{j}\right), i, j=1,2, \ldots$, we have

$$
\begin{aligned}
\lim _{i \rightarrow \infty} \frac{1}{\left\|f\left(x_{i}\right)\right\|} f\left(x_{i}\right) & =\lim _{i \rightarrow \infty} \frac{1}{\sqrt{y_{i}^{2}+\left(a+b k_{i}^{2} \cos k_{i}\right)^{2}}}\left[\begin{array}{c}
-\operatorname{sign}\left(y_{i}\right)\left(a+b k_{i}^{2} \cos k_{i}\right) \\
\left|y_{i}\right|
\end{array}\right] \\
& =\left[\begin{array}{c}
-\operatorname{sign}\left(y_{1}\right) \operatorname{sign}\left(a+b k^{2} \cos k\right) \\
0
\end{array}\right] \\
& \in\left\{\left[\begin{array}{ll} 
\pm 1 & 0
\end{array}\right]^{\mathrm{T}}\right\} .
\end{aligned}
$$

Thus, for every $x \in \mathcal{N}_{0} \backslash \mathcal{Z}, \mathcal{L}_{x}$ is finite. By Remark 4.3, for every $x \in \mathcal{N}_{0} \backslash \mathcal{Z}$, $\mathcal{F}_{x}=\operatorname{coco} \mathcal{L}_{x}=\{(c, 0): c \in \mathbb{R}\}$. On the other hand, for every $x \in \mathcal{N}_{0}, T_{x} \mathcal{N}_{0}=$ $\{(0, c): c \in \mathbb{R}\}$. Hence $f$ is nontangent to $\mathcal{N}_{0}$ at every point $x \in \mathcal{N}_{0} \backslash \mathcal{Z}$.

In order to apply Theorem 5.1, we note that, in the notation of Theorem 5.1, $\mathcal{N}_{1} \subseteq \mathcal{M}_{0} \subseteq \mathcal{Z}$. If $\mathcal{N}_{1}$ is empty, then $\mathcal{M}_{1} \subseteq \mathcal{N}_{1}$ is empty. If $\mathcal{N}_{1}$ is nonempty, $\mathcal{Z}$ consists only of isolated points and hence $T_{x} \mathcal{N}_{1}=\{0\}$ for every $x \in \mathcal{N}_{1}$. Consequently, $f$ is nontangent to $\mathcal{N}_{1}$ at every point in $\mathcal{N}_{1}$ and hence $\mathcal{M}_{1}$ is empty. In either case, $\mathcal{M}_{1} \subseteq \mathcal{S}$ vacuously. It now follows from Theorem 5.1 that the system (10)-(11) is convergent relative to $\mathbb{R}^{2}$.

The following two results follow easily from Theorem 5.1.

Corollary 5.1. Suppose $\mathcal{O}_{x}$ is relatively bounded in $\mathcal{G}$ for all $x \in \mathcal{G}$, and assume that there exists a continuous function $V: \mathcal{G} \rightarrow \mathbb{R}$ such that $\dot{V}$ is defined on $\mathcal{G}$ and satisfies $\dot{V}(x) \leq 0$ for all $x \in \mathcal{G}$. Let $\mathcal{N}$ be the largest invariant subset of $\dot{V}^{-1}(0)$. If $f$ is nontangent to $\mathcal{N}$ at every $z \in \mathcal{N}$ relative to $\mathcal{G}$, then the system (6) is convergent relative to $\mathcal{G}$.

Proof. Suppose $f$ is nontangent to $\mathcal{N}$ at every $z \in \mathcal{N}$ relative to $\mathcal{G}$. In the notation of Theorem 5.1, $\mathcal{N}_{0}=\mathcal{N}$, while $\mathcal{M}_{0}=\varnothing$. Thus $\mathcal{M}_{0} \subseteq \mathcal{S}$ vacuously, and the result follows from Theorem 5.1.

Corollary 5.2. Suppose $\mathcal{O}_{x}$ is relatively bounded in $\mathcal{G}$ for all $x \in \mathcal{G}$, and assume that there exists a continuous function $V: \mathcal{G} \rightarrow \mathbb{R}$ such that $\dot{V}$ is defined on $\mathcal{G}$ and satisfies $\dot{V}(x) \leq 0$ for all $x \in \mathcal{G}$. Let $\mathcal{N}$ be the largest invariant subset of $\dot{V}^{-1}(0)$. If every point in $\mathcal{N}$ is Lyapunov stable relative to $\mathcal{G}$, then the system (6) is convergent relative to $\mathcal{G}$.

Proof. Suppose every point in $\mathcal{N}$ is Lyapunov stable relative to $\mathcal{G}$. Then, in the notation of Theorem 5.1, $\mathcal{M}_{0} \subseteq \mathcal{N}_{0}=\mathcal{N}=\mathcal{S}$. The result now follows from Theorem 5.1 .

In the following example, we illustrate Corollary 5.1 by applying it to the system considered in Example 1.1.

Example 5.4. Consider the system described in Example 1.1. Suppose $\alpha \leq \beta$, and let $\mathcal{G}=\mathbb{R}^{2}$. Consider the Lyapunov function $V: \mathcal{G} \rightarrow \mathbb{R}$ given by $V(x)=$ $\frac{1}{4}\left(x_{1}^{2}+x_{2}^{2}-1\right)^{2}$. It is easy to compute $\dot{V}(x)=-\left(x_{1}^{2}+x_{2}^{2}\right)\left|x_{1}^{2}+x_{2}^{2}-1\right|^{1+\alpha}$ so that $\dot{V}(x) \leq 0$ for all $x \in \mathcal{G}$ and $\dot{V}^{-1}(0)=\mathrm{S}^{1} \cup\{0\}$. Since every point in $\dot{V}^{-1}(0)$ is an equilibrium, the largest invariant subset of $\dot{V}^{-1}(0)$ is $\mathcal{N}=\dot{V}^{-1}(0)$. We know from Example 4.2 that $f$ is nontangent to $\mathcal{N}$ at every point in $S^{1}$ relative to $\mathcal{G}$. Since $\{0\}$ is an isolated point of $\mathcal{N}$, it follows that $f$ is nontangent to $\mathcal{N}$ at 0 relative to $\mathcal{G}$. Thus the hypotheses of Corollary 5.1 are satisfied, and we conclude that the system considered in Example 1.1 is convergent relative to $\mathcal{G}=\mathbb{R}^{2}$ in the case where $\alpha \leq \beta$. 
6. Restricted prolongations, Lyapunov stability, and nontangency. In this section, we develop three key ideas that will be needed for the nontangencybased Lyapunov tests for Lyapunov stability, semistability, and asymptotic stability that we present in the next section. The first of these ideas, given in Proposition 6.2, is that an equilibrium is Lyapunov stable if and only if its restricted prolongation, as defined below, is a singleton set. The second key idea, given in Proposition 6.3, is to use Proposition 4.1 to show that the restricted prolongation of an equilibrium of (6) is a singleton set if and only if the vector field $f$ is nontangent to the restricted prolongation at the equilibrium. Since it is not generally possible to find the restricted prolongation of an equilibrium in practice, nontangency of the vector field $f$ to the restricted prolongation is difficult to verify in applications. Since nontangency to any outer estimate of the restricted prolongation implies nontangency to the restricted prolongation itself, the third key idea is to determine outer estimates of the restricted prolongation that are easier to find in practice. Proposition 6.4 gives outer estimates of restricted prolongations in terms of connected components of invariant and negatively invariant subsets of level and sublevel sets of a Lyapunov function and its derivative. This result depends on the invariance properties of restricted prolongations given in Proposition 6.1 .

Given a point $x \in \mathcal{G}$ and a relatively open and relatively bounded neighborhood $\mathcal{U} \subseteq \mathcal{G}$ of $x$, the restricted prolongation of $x$ with respect to $\mathcal{U}$ is the set $\mathcal{R}_{x}^{\mathcal{U}} \subseteq \overline{\mathcal{U}}$ of all subsequential limits of sequences of the form $\left\{\psi\left(t_{i}, x_{i}\right)\right\}$, where $\left\{t_{i}\right\}$ is a sequence in $[0, \infty)$ and $\left\{x_{i}\right\}$ is a sequence in $\mathcal{U}$ converging to $x$ such that the set $\psi\left(\left[0, t_{i}\right] \times\left\{x_{i}\right\}\right)$ is contained in $\overline{\mathcal{U}}$ for every $i$. It is easy to see that, for every $x \in \mathcal{G}$ and every relatively bounded and open neighborhood $\mathcal{U} \subseteq \mathcal{G}$ of $x, \mathcal{R}_{x}^{\mathcal{U}}$ contains $x$ and is thus nonempty. The restricted prolongation of $x$ relative to $\mathcal{G}$ is the union $\mathcal{R}_{x}$ of all sets of the form $\mathcal{R}_{x}^{\mathcal{U}}$ where $\mathcal{U} \subseteq \mathcal{G}$ is a relatively open and relatively bounded neighborhood of $x$. It can be shown that $\mathcal{R}_{x}$ is the set of all subsequential limits of sequences of the form $\left\{\psi\left(t_{i}, x_{i}\right)\right\}$, where $\left\{t_{i}\right\}$ is a sequence in $[0, \infty)$ and $\left\{x_{i}\right\}$ is a sequence in $\mathcal{G}$ converging to $x$ such that the set $\cup_{i} \psi\left(\left[0, t_{i}\right] \times\left\{x_{i}\right\}\right)$ is relatively bounded in $\mathcal{G}$. The restricted prolongation is a subset of the positive prolongation as defined in $[7,8]$.

The following result gives invariance properties of restricted prolongations.

Proposition 6.1. Suppose $x \in \mathcal{G}$, and let $\mathcal{U} \subseteq \mathcal{G}$ be a relatively open and relatively bounded neighborhood of $x$. Then $\mathcal{R}_{x}^{\mathcal{U}}$ and $\mathcal{R}_{x}$ are connected. Moreover, if $x$ is an equilibrium, then $\mathcal{R}_{x}^{\mathcal{U}}$ is negatively invariant and $\mathcal{R}_{x}$ is invariant.

Proof. To prove the first part of the proposition, suppose $\mathcal{R}_{x}^{\mathcal{U}}$ is not connected. Then there exist open and disjoint subsets $\mathcal{V}$ and $\mathcal{W}$ of $\mathbb{R}^{n}$ such that $\mathcal{R}_{x}^{\mathcal{U}} \subseteq \mathcal{V} \cup \mathcal{W}$, $\mathcal{R}_{x}^{\mathcal{U}} \cap \mathcal{V} \neq \varnothing$, and $\mathcal{R}_{x}^{\mathcal{U}} \cap \mathcal{W} \neq \varnothing$. Since $x \in \mathcal{R}_{x}^{\mathcal{U}}$, we may assume without loss of generality that $x \in \mathcal{V}$.

Consider $z \in \mathcal{R}_{x}^{\mathcal{U}} \cap \mathcal{W}$. There exist a sequence $\left\{x_{i}\right\}$ in $\mathcal{G}$ converging to $x$ and a sequence $\left\{t_{i}\right\}$ in $[0, \infty)$ such that $\lim _{i \rightarrow \infty} \psi\left(t_{i}, x_{i}\right)=z$ and $\psi\left(\left[0, t_{i}\right] \times\left\{x_{i}\right\}\right) \subseteq \overline{\mathcal{U}}$ for every $i$. Since $\lim _{i \rightarrow \infty} \psi\left(0, x_{i}\right)=x$ and $\lim _{i \rightarrow \infty} \psi\left(t_{i}, x_{i}\right)=z$, there exists $k>0$ such that, for every $i>k$, the connected set $\psi\left(\left[0, t_{i}\right] \times\left\{x_{i}\right\}\right)$ intersects the disjoint, relatively open sets $\mathcal{V}$ and $\mathcal{W}$. We conclude that, for every $i>k$, there exists $\tau_{i} \in\left[0, t_{i}\right]$ such that $\psi\left(\tau_{i}, x_{i}\right) \in(\mathcal{V} \cup \mathcal{W})^{\mathrm{c}}=\mathcal{V}^{\mathrm{c}} \cap \mathcal{W}^{\mathrm{c}}$, where $\mathcal{A}^{\mathrm{c}}$ denotes the complement $\mathbb{R}^{n} \backslash \mathcal{A}$ of the set $\mathcal{A} \subseteq \mathbb{R}^{n}$. The sequence $\left\{\psi\left(\tau_{i}, x_{i}\right)\right\}$ is relatively bounded in $\mathcal{G}$ and contained in the closed set $\mathcal{V}^{\mathrm{c}} \cap \mathcal{W}^{\mathrm{c}}$. Therefore, the sequence $\left\{\psi\left(\tau_{i}, x_{i}\right)\right\}$ has a subsequential limit in $\mathcal{V}^{\mathrm{c}} \cap \mathcal{W}^{\mathrm{c}}$. However, by definition, every subsequential limit of the sequence $\left\{\psi\left(\tau_{i}, x_{i}\right)\right\}$ is contained in $\mathcal{R}_{x}^{\mathcal{U}} \subset \mathcal{V} \cup \mathcal{W}$. This contradiction proves that $\mathcal{R}_{x}^{\mathcal{U}}$ is connected. 
It now follows that $\mathcal{R}_{x}$ is a union of connected sets of the form $\mathcal{R}_{x}^{\mathcal{U}}$, all of which contain $x$, and is thus connected [10, Thm. V.1.5].

To prove the second part of the proposition, suppose $x \in \mathcal{G}$ is an equilibrium, and consider $z \in \mathcal{R}_{x}^{\mathcal{U}}$. There exist a sequence $\left\{t_{i}\right\}$ in $[0, \infty)$ and a sequence $\left\{x_{i}\right\}$ in $\mathcal{G}$ converging to $x$ such that $z=\lim _{i \rightarrow \infty} \psi\left(t_{i}, x_{i}\right)$ and, for every $i, \psi\left(h, x_{i}\right) \in \overline{\mathcal{U}}$ for every $h \in\left[0, t_{i}\right]$.

Now, let $t \geq 0$. If $z=x$, then $\psi(\tau, x)=x=z$ for every $\tau \in[0, t]$. Hence suppose $z \neq x$. If the sequence $\left\{t_{i}\right\}$ has a bounded subsequence, say, $\left\{t_{i_{k}}\right\}$, then we may assume that $\lim _{k \rightarrow \infty} t_{i_{k}}=T$ so that $z=\lim _{k \rightarrow \infty} \psi\left(t_{i_{k}}, x_{i_{k}}\right)=\psi(T, x)=x$. However, this contradicts our assumption that $z \neq x$. Hence we conclude that the sequence $\left\{t_{i}\right\}$ diverges. Therefore, there exists $N>0$ such that $t_{i}>t$ for all $i>N$. The sequence $\left\{\psi\left(t_{i+N}-t, x_{i+N}\right)\right\}_{i=1}^{\infty}$ is contained in $\overline{\mathcal{U}}$ and hence relatively bounded in $\mathcal{G}$. Let $y \in \mathcal{G}$ be a subsequential limit point of this sequence. Clearly, $y \in \mathcal{R}_{x}^{\mathcal{U}}$. Also, by continuity and the semigroup property, $\psi(t, y)=z$, and, for every $\tau \in[0, t], \psi(\tau, y)$ is a subsequential limit of the bounded sequence $\left\{\psi\left(t_{i+N}+\tau-t, x_{i+N}\right)\right\}_{i=1}^{\infty}$ and hence contained in $\mathcal{R}_{x}^{\mathcal{U}}$. This proves the negative invariance of $\mathcal{R}_{x}^{\mathcal{U}}$.

From the negative invariance of $\mathcal{R}_{x}^{\mathcal{U}}$, it follows that $\mathcal{R}_{x}$ is the union of negatively invariant sets and hence negatively invariant. To prove positive invariance of $\mathcal{R}_{x}$, let $\tau \geq 0$ and $z \in \mathcal{R}_{x}$. There exist a sequence $\left\{t_{i}\right\}$ in $[0, \infty)$ and a sequence $\left\{x_{i}\right\}$ in $\mathcal{G}$ converging to $x$ and a compact set $\mathcal{M} \subseteq \mathcal{G}$ such that $z=\lim _{i \rightarrow \infty} \psi\left(t_{i}, x_{i}\right)$ and, for every $i$ and every $h \in\left[0, t_{i}\right], \psi\left(h, x_{i}\right) \in \mathcal{M}$. Now, for every $i$ and every $h \in\left[0, t_{i}+\tau\right]$, $\psi\left(h, x_{i}\right)$ is contained in the compact subset $\psi([0, \tau] \times \mathcal{M})$ of $\mathcal{G}$. Hence a subsequence of the sequence $\left\{\psi\left(t_{i}+\tau, x_{i}\right)\right\}$ converges in $\mathcal{G}$. The limit of this subsequence is $\psi(\tau, z)$ by continuity and the semigroup property and is contained in $\mathcal{R}_{x}$ by definition. Thus $\psi(\tau, z) \in \mathcal{R}_{x}$ for every $z \in \mathcal{R}_{x}$ and $\tau \geq 0$. This proves the positive invariance and hence the invariance of $\mathcal{R}_{x}$.

The utility of prolongations in stability analysis stems from the well-known fact that an equilibrium point is Lyapunov stable if and only if the positive prolongation of the equilibrium consists only of the equilibrium point. See, for instance, [7, Prop. 7.3] and [8, Thm. V.1.12]. We prove the same result for restricted prolongations in Proposition 6.2 below. Since, as mentioned above, the restricted prolongation of a point is a subset of the positive prolongation of the point, Proposition 6.2 is a sharper version of the results [7, Prop. 7.3] and [8, Thm. V.1.12]. However, our reason for considering restricted prolongations instead of positive prolongations in this paper is that restricted prolongations are invariant and connected as proved in Proposition 6.1 above. Positive prolongations, on the other hand, may neither be connected nor invariant under our assumptions on the system (6). Since the invariance and connectedness of restricted prolongations play a crucial role in our main results, we introduce restricted prolongations instead of using positive prolongations.

Proposition 6.2. Suppose $x \in \mathcal{G}$, and let $\mathcal{U} \subseteq \mathcal{G}$ be a relatively open and relatively bounded neighborhood of $x$. Then the following statements are equivalent.

(i) The point $x$ is a Lyapunov stable equilibrium relative to $\mathcal{G}$.

(ii) $\mathcal{R}_{x}^{\mathcal{U}}=\{x\}$.

(iii) $\mathcal{R}_{x}=\{x\}$.

Proof. If $x$ is a Lyapunov stable equilibrium relative to $\mathcal{G}$, then the positive prolongation of $x$, which contains $\mathcal{R}_{x}$, is $\{x\}$ [7, Prop. 7.3], [8, Th. V.1.12], and hence $\mathcal{R}_{x}=\{x\}$. Thus (i) implies (iii).

Since $x \in \mathcal{R}_{x}^{\mathcal{U}}$ and $\mathcal{R}_{x}^{\mathcal{U}} \subseteq \mathcal{R}_{x}$, (iii) implies (ii).

To show that (ii) implies (i), suppose $x$ is not a Lyapunov stable equilibrium 
relative to $\mathcal{G}$. Then there exist a neighborhood $\mathcal{V} \subseteq \mathcal{U}$ of $x$ that is relatively bounded and relatively open in $\mathcal{G}$, a sequence $\left\{x_{i}\right\}$ in $\mathcal{V}$ converging to $x$, and a sequence $\left\{t_{i}\right\}$ in $[0, \infty)$ such that $\psi\left(t_{i}, x_{i}\right) \in$ bd $\mathcal{V}$ for every $i$. Without loss of generality, we may assume that the sequence $\left\{t_{i}\right\}$ is chosen such that, for every $i, \psi\left(h, x_{i}\right) \in \mathcal{V}$ for all $h \in$ $\left[0, t_{i}\right)$. Now, every subsequential limit of the relatively bounded sequence $\left\{\psi\left(t_{i}, x_{i}\right)\right\}$ is distinct from $x$ by construction and is contained in $\mathcal{R}_{x}^{\mathcal{U}}$ by definition. Thus the negation of (i) implies the negation of (ii). Hence it follows that (ii) implies (i).

The following result characterizes Lyapunov stable equilibrium points in terms of nontangency.

Proposition 6.3. Let $x \in \mathcal{G}$, and let $\mathcal{U} \subseteq \mathcal{G}$ be a relatively open and relatively bounded neighborhood of $x$. Then the following statements are equivalent.

(i) The point $x$ is a Lyapunov stable equilibrium relative to $\mathcal{G}$.

(ii) The vector field $f$ is nontangent to $\mathcal{R}_{x}^{\mathcal{U}}$ at $x$ relative to $\mathcal{G}$.

(iii) The vector field $f$ is nontangent to $\mathcal{R}_{x}$ at $x$ relative to $\mathcal{G}$.

Proof. If $x$ is a Lyapunov stable equilibrium relative to $\mathcal{G}$, then it follows from Proposition 6.2 that $\mathcal{R}_{x}=\{x\}$ and hence $T_{x} \mathcal{R}_{x}=\{0\}$. Thus (i) implies (iii).

Since $\mathcal{R}_{x}^{\mathcal{U}} \subseteq \mathcal{R}_{x}$, it follows that $T_{x} \mathcal{R}_{x}^{\mathcal{U}} \subseteq T_{x} \mathcal{R}_{x}$ and hence (iii) implies (ii).

Now, suppose (ii) holds so that $T_{x} \mathcal{R}_{x}^{\mathcal{U}} \cap \mathcal{F}_{x} \subseteq\{0\}$. Let $z \in \mathcal{R}_{x}^{\mathcal{U}}$. There exist a sequence $\left\{x_{i}\right\}$ converging to $x$ and a sequence $\left\{t_{i}\right\}$ in $[0, \infty)$ such that $\cup_{i} \psi\left(\left[0, t_{i}\right] \times\right.$ $\left.\left\{x_{i}\right\}\right) \subset \overline{\mathcal{U}}$ and $\lim _{i \rightarrow \infty} \psi\left(t_{i}, x_{i}\right)=z$.

First, suppose that the sequence $\left\{t_{i}\right\}$ converges to 0 . Then by continuity, $z=$ $\lim _{i \rightarrow \infty} \psi\left(t_{i}, x_{i}\right)=\psi(0, x)=x$. Next, suppose the sequence $\left\{t_{i}\right\}$ does not converge to 0 . Then there exists a subsequence $\left\{t_{i_{k}}\right\}$ of the sequence $\left\{t_{i}\right\}$ such that $\inf _{k} t_{i_{k}}>0$. Let $\mathcal{I}_{k}=\left[0, t_{i_{k}}\right]$ for each $k$, and let $\mathcal{B} \subseteq \overline{\mathcal{U}}$ denote the set of all subsequential limits of sequences of the form $\left\{\psi\left(\tau_{k}, x_{i_{k}}\right)\right\}_{k=1}^{\infty}$, where $\tau_{k} \in \mathcal{I}_{k}$ for every $k$. By construction, $z \in \mathcal{B}$ and $\mathcal{B} \subseteq \mathcal{R}_{x}^{\mathcal{U}}$. Therefore, $T_{x} \mathcal{B} \cap \mathcal{F}_{x} \subseteq T_{x} \mathcal{R}_{x}^{\mathcal{U}} \cap \mathcal{F}_{x} \subseteq\{0\}$; that is, $f$ is nontangent to $\mathcal{B}$ at $x$ relative to $\mathcal{G}$. It now follows from Proposition 4.1 that $\mathcal{B}=\{x\}$. Hence $z=x$. Since $z \in \mathcal{R}_{x}^{\mathcal{U}}$ was arbitrary, it follows that $\mathcal{R}_{x}^{\mathcal{U}}=\{x\}$. Proposition 6.2 now implies that (i) holds.

Example 6.1. Consider the system described in Example 1.1, and assume that $\alpha \geq \beta+1$. Let $\mathcal{G}=\mathbb{R}^{2}$. As discussed in Example 1.1, every point in $\mathrm{S}^{1}$ is an unstable equilibrium. From Figure 1, we observe that, for every $z \in S^{1}, \mathcal{R}_{z}=S^{1}$. Since $S^{1}$ consists only of equilibrium points, it follows that $\mathcal{R}_{z}$ is invariant for every $z \in \mathrm{S}^{1}$. This illustrates Proposition 6.1. As seen in Example 4.2, the vector field $f$ is not nontangent to $S^{1}$ at any $z \in S^{1}$, thus illustrating the equivalence of (i) and (iii) in Proposition 6.3.

As mentioned earlier, it is not generally possible to find the restricted prolongation of an equilibrium, and hence Proposition 6.3 cannot be directly used in applications. However, the following proposition shows that Lyapunov functions can be used to obtain sets that contain the restricted prolongation of an equilibrium. The proof uses the properties of invariance and connectedness of restricted prolongations given in Proposition 6.1.

Proposition 6.4. Suppose $V: \mathcal{G} \rightarrow \mathbb{R}$ is a continuous function such that $\dot{V}$ is defined on $\mathcal{G}$. Let $x \in \mathcal{G}$ be an equilibrium, and let $\mathcal{W} \subseteq \mathcal{G}$ be a relatively open neighborhood of $x$. Let $\mathcal{M}_{x}$ and $\mathcal{N}_{x}$ denote the largest connected subsets of $V^{-1}(V(x)) \cap \mathcal{W}$ and $\overline{\dot{V}^{-1}(0)} \cap \mathcal{W}$, respectively, that contain $x$ and are negatively invariant. Let $\mathcal{P}_{x}$ denote the largest connected subset of $\{z \in \mathcal{G}: V(z) \leq V(x)\}$ that contains $x$ and is invariant. Then the following statements hold.

(i) If $x$ is a global maximizer of $\dot{V}$ relative to $\mathcal{G}$, then $\mathcal{R}_{x} \subseteq \mathcal{P}_{x}$. 
(ii) If $x$ is a local maximizer of $\dot{V}$ relative to $\mathcal{G}$ and a local minimizer of $V$ relative to the set $\mathcal{K} \stackrel{\text { def }}{=} \mathcal{G} \backslash \overline{\dot{V}^{-1}(0)}$, then there exists a relatively open and relatively bounded neighborhood $\mathcal{V} \subseteq \mathcal{W}$ of $x$ such that $\mathcal{R}_{x}^{\mathcal{V}} \subseteq \mathcal{M}_{x}$.

(iii) $\mathcal{M}_{x} \subseteq \mathcal{N}_{x}$.

Proof. (i) Suppose $x$ is a global maximizer of $\dot{V}$ relative to $\mathcal{G}$ so that $\dot{V}(z) \leq$ $\dot{V}(x)=0$ for every $z \in \mathcal{G}$. Consider $z \in \mathcal{R}_{x}$. Let $\left\{x_{i}\right\}$ be a sequence in $\mathcal{G}$ converging to $x$ and $\left\{t_{i}\right\}$ a sequence in $[0, \infty)$ such that $\lim _{i \rightarrow \infty} \psi\left(t_{i}, x_{i}\right)=z$. Since $V$ is continuous and decreasing along the solutions of (6), it follows that $V(z)=\lim _{i \rightarrow \infty} V\left(\psi\left(t_{i}, x_{i}\right)\right) \leq$ $\lim _{i \rightarrow \infty} V\left(x_{i}\right)=V(x)$. Thus $\mathcal{R}_{x}$ is contained in the set $\{z \in \mathcal{G}: V(z) \leq V(x)\}$. By Proposition $6.1, \mathcal{R}_{x}$ is invariant and connected. Also, $x \in \mathcal{R}_{x}$. Hence it follows that $\mathcal{R}_{x} \subseteq \mathcal{P}_{x}$.

(ii) Let $x$ be a local maximizer of $\dot{V}$ relative to $\mathcal{G}$ and a local minimizer of $V$ relative to the set $\mathcal{K}$. Let $\mathcal{U} \subseteq \mathcal{W}$ be a relatively open neighborhood of $x$ such that $\dot{V}(z) \leq \dot{V}(x)=0$ for every $z \in \mathcal{U}$ and $V(x) \leq V(w)$ for every $w \in \mathcal{U} \cap \mathcal{K}$. Consider $w \in$ $\mathcal{U} \cap \overline{\mathcal{K}}$. There exists a sequence $\left\{w_{i}\right\}$ in $\mathcal{U} \cap \mathcal{K}$ that converges to $w$. Then $V\left(w_{i}\right) \geq V(x)$ for every $i$. It now follows by continuity of $V$ that $V(w)=\lim _{i \rightarrow \infty} V\left(w_{i}\right) \geq V(x)$. Next let $\mathcal{V} \subseteq \mathcal{G}$ be a relatively open and relatively bounded neighborhood of $x$ such that $\overline{\mathcal{V}} \subseteq \mathcal{U}$. Then, for every $w \in \overline{\mathcal{V}} \cap \overline{\mathcal{K}}, V(w) \geq V(x)$.

Now consider $z \in \mathcal{R}_{x}^{\mathcal{V}}$. Let $\left\{x_{i}\right\}$ be a sequence in $\mathcal{V}$ converging to $x$ and $\left\{t_{i}\right\}$ a sequence in $[0, \infty)$ such that the sequence $\left\{\psi\left(t_{i}, x_{i}\right)\right\}$ converges to $z$ and, for every $i, \psi\left(\tau, x_{i}\right) \in \overline{\mathcal{V}} \subset \mathcal{U}$ for every $\tau \in\left[0, t_{i}\right]$. Then, $V(z)=\lim _{i \rightarrow \infty} V\left(\psi\left(t_{i}, x_{i}\right)\right) \leq$ $\lim _{i \rightarrow \infty} V\left(x_{i}\right)=V(x)$. Thus $V(z) \leq V(x)$. We claim that $V(z)=V(x)$. To see this, first suppose $z \in \mathcal{R}_{x}^{\mathcal{V}} \cap \overline{\mathcal{K}} \subseteq \overline{\mathcal{V}} \cap \overline{\mathcal{K}}$. Then, since $V(w) \geq V(x)$ for every $w \in \overline{\mathcal{V}} \cap \overline{\mathcal{K}}$, it

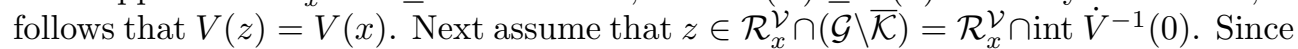
$\mathcal{G} \backslash \overline{\mathcal{K}}$ is relatively open in $\mathcal{G}$ and $z \in \mathcal{G} \backslash \overline{\mathcal{K}}$, there exists $m>0$ such that $\psi\left(t_{i}, x_{i}\right) \in \mathcal{G} \backslash \overline{\mathcal{K}}$ for every $i>m$. For each $i>m$, let $\tau_{i}$ denote the smallest number in $\left[0, t_{i}\right]$ such that $\psi\left(t, x_{i}\right) \in \mathcal{G} \backslash \overline{\mathcal{K}}$ for all $t \in\left(\tau_{i}, t_{i}\right]$. The sequence $\left\{\tau_{i}\right\}_{i=m}^{\infty}$ either has a positive subsequence or is eventually zero.

First consider the case where $\left\{\tau_{i}\right\}_{i=m}^{\infty}$ has a positive subsequence, say, $\left\{\tau_{i_{k}}\right\}_{k=1}^{\infty}$. By the continuity of $\psi$, it follows that $\psi\left(\tau_{i_{k}}, x_{i_{k}}\right) \in \overline{\mathcal{K}}$ for every $k$. Since $V$ is nonincreasing along the solutions of (6) in $\mathcal{U}, V\left(\psi\left(\tau_{i_{k}}, x_{i_{k}}\right)\right) \leq V\left(x_{i_{k}}\right)$ for all $k$, and hence $\lim _{k \rightarrow \infty} V\left(\psi\left(\tau_{i_{k}}, x_{i_{k}}\right)\right) \leq \lim _{k \rightarrow \infty} V\left(x_{i_{k}}\right)=V(x)$. On the other hand, $x$ is a global minimizer of $V$ on the set $\overline{\mathcal{V}} \cap \overline{\mathcal{K}}$ so that $V\left(\psi\left(\tau_{i_{k}}, x_{i_{k}}\right)\right) \geq V(x)$ for all $k$. Thus $\lim _{k \rightarrow \infty} V\left(\psi\left(\tau_{i_{k}}, x_{i_{k}}\right)\right)=V(x)$ for all $k$. Since $\psi\left(t, x_{i}\right) \in \mathcal{G} \backslash \overline{\mathcal{K}} \subseteq \dot{V}^{-1}(0)$ for all $t \in\left(\tau_{i}, t_{i}\right]$ and every $i$, it follows that $V\left(\psi\left(t_{i_{k}}, x_{i_{k}}\right)\right)=V\left(\psi\left(\tau_{i_{k}}, x_{i_{k}}\right)\right)$ for all $k$. Hence $V(z)=\lim _{k \rightarrow \infty} V\left(\psi\left(t_{i_{k}}, x_{i_{k}}\right)\right)=\lim _{k \rightarrow \infty} V\left(\psi\left(\tau_{i_{k}}, x_{i_{k}}\right)\right)=V(x)$.

Finally, consider the case where the sequence $\left\{\tau_{i}\right\}_{i=m}^{\infty}$ is eventually zero. In this case, there exists $K>0$ such that, for every $i>K$ and every $t \in\left[0, t_{i}\right]$, it follows that $V\left(\psi\left(t, x_{i}\right)\right)=V\left(x_{i}\right)$. Hence $V(z)=\lim _{i \rightarrow \infty} V\left(\psi\left(t_{i}, x_{i}\right)\right)=\lim _{i \rightarrow \infty} V\left(x_{i}\right)=V(x)$.

We have thus shown that if $z \in \mathcal{R}_{x}^{\mathcal{V}}$, then $V(z)=V(x)$. Therefore, $\mathcal{R}_{x}^{\mathcal{V}} \subseteq$ $V^{-1}(V(x)) \cap \overline{\mathcal{V}} \subset V^{-1}(V(x)) \cap \mathcal{W}$. By Proposition $6.1, \mathcal{R}_{x}^{\mathcal{V}}$ is negatively invariant and connected, and $x \in \mathcal{R}_{x}^{\mathcal{V}}$. Hence $\mathcal{R}_{x}^{\mathcal{V}} \subseteq \mathcal{M}_{x}$.

(iii) Consider $z \in \mathcal{M}_{x}$, and let $t>0$. By negative invariance, there exists $w \in \mathcal{M}_{x}$ such that $\psi(t, w)=z$ and $\psi(\tau, w) \in \mathcal{M}_{x} \subseteq V^{-1}(V(x))$ for all $\tau \in[0, t]$. Hence $V(\psi(\tau, w))=V(x)$ for every $\tau \in[0, t]$, and thus $\dot{V}(\psi(\tau, w))=0$ for every $\tau \in[0, t)$. Let $\left\{t_{i}\right\}$ be a sequence in $[0, t)$ converging to $t$. Then, by the continuity of $\psi,\left\{\psi\left(t_{i}, w\right)\right\}$ is a sequence in $\dot{V}^{-1}(0)$ that converges to $z$. It follows that $z \in \overline{\dot{V}^{-1}(0)}$. Thus $\mathcal{M}_{x} \subseteq \overline{\dot{V}^{-1}(0)}$. Since $\mathcal{M}_{x}$ is negatively invariant, connected, contains $x$, and is contained in $\mathcal{W}$, the result follows. 
It is interesting to note the parallels between the ideas used in this section and those used in the previous section. The propositions given in section 5 allow us to conclude convergence of a solution of (6) under the assumption of nontangency of the vector field $f$ to an outer estimate of the positive limit set of that solution. The results of this section allow us to conclude the Lyapunov stability of an equilibrium of (6) under the assumption of nontangency of the vector field $f$ to an outer estimate of the restricted prolongation of the equilibrium. The outer estimates of the positive limit set in section 5 and the restricted prolongation in this section are in terms of invariant and negatively invariant subsets of level and sublevel sets of a Lyapunov function and its derivative. Moreover, the results of sections 5 and 6 depend on the invariance properties of the positive limit set and the restricted prolongation, respectively.

7. Stability theorems. In this section, we use the results of the previous two sections to derive Lyapunov results for Lyapunov stability, semistability, and asymptotic stability. The main results are Theorems 7.1 and 7.2. These results do not make any assumptions about the sign definiteness of the Lyapunov function. Instead, these results require only that the Lyapunov function derivative be nonpositive and the equilibrium be a local minimizer of the Lyapunov function on the set of points where the Lyapunov function derivative is negative. The weaker assumptions on the Lyapunov function are supplemented by assuming nontangency of the vector field to the level set of the Lyapunov function containing the equilibrium or to the closure of the zero-level set of the Lyapunov function derivative. In both Theorems 7.1 and 7.2, Propositions 6.4 and 5.3 are used to "trap" the restricted prolongation and the positive limit set, respectively, in the level sets of the Lyapunov function and its derivative. Propositions 6.3 and 5.2 are then used to deduce stability and convergence from nontangency.

TheOREM 7.1. Suppose $V: \mathcal{G} \rightarrow \mathbb{R}$ is a continuous function such that $\dot{V}$ is defined on $\mathcal{G}$. Let $x \in \mathcal{E}$ be a local maximizer of $\dot{V}$ relative to $\mathcal{G}$ and a local minimizer of $V$ relative to the set $\mathcal{K} \stackrel{\text { def }}{=} \mathcal{G} \backslash \overline{\dot{V}^{-1}(0)}$. Let $\mathcal{W} \subseteq \mathcal{G}$ be a relatively open neighborhood of $x$. For every $z \in \mathcal{E} \cap \mathcal{W}$, let $\mathcal{M}_{z}$ denote the largest connected subset of $V^{-1}(V(z)) \cap \mathcal{W}$ that is negatively invariant and contains $z$. Let $\mathcal{N}$ denote the largest negatively invariant subset of $\overline{\dot{V}^{-1}(0)} \cap \mathcal{W}$ and, for every $z \in \mathcal{N}$, let $\mathcal{N}_{z}$ denote the connected component of $\mathcal{N}$ containing $z$.

Then the following statements hold.

(i) If $f$ is nontangent to $\mathcal{M}_{x}$ at $x$ relative to $\mathcal{G}$, then $x$ is Lyapunov stable relative to $\mathcal{G}$.

(ii) If $f$ is nontangent to $\mathcal{N}_{x}$ at $x$ relative to $\mathcal{G}$, then $x$ is Lyapunov stable relative to $\mathcal{G}$.

(iii) If there exists a relatively open neighborhood $\mathcal{U} \subseteq \mathcal{W}$ of $x$ such that every equilibrium in $\mathcal{U}$ is a local minimizer of $V$ relative to $\mathcal{K}$ and, for every $z \in$ $\mathcal{N} \cap \mathcal{U}, f$ is nontangent to $\mathcal{N}_{z}$ at $z$ relative to $\mathcal{G}$, then $x$ is semistable relative to $\mathcal{G}$.

(iv) If $x$ is an isolated equilibrium, and there exists a relatively open neighborhood $\mathcal{U} \subseteq \mathcal{W}$ of $x$ such that, for every $z \in \mathcal{N} \cap \mathcal{U}, f$ is nontangent to $\mathcal{N}_{z}$ at $z$ relative to $\mathcal{G}$, then $x$ is asymptotically stable relative to $\mathcal{G}$.

Proof. (i) Suppose $T_{x} \mathcal{M}_{x} \cap \mathcal{F}_{x} \subseteq\{0\}$. By (ii) of Proposition 6.4, there exists a relatively open and bounded neighborhood $\mathcal{V} \subseteq \mathcal{W}$ of $x$ such that $\mathcal{R}_{x}^{\mathcal{V}} \subseteq \mathcal{M}_{x}$. Therefore, $T_{x} \mathcal{R}_{x}^{\mathcal{V}} \cap \mathcal{F}_{x} \subseteq T_{x} \mathcal{M}_{x} \cap \mathcal{F}_{x} \subseteq\{0\}$. Hence, by Proposition 6.3, $x$ is a Lyapunov stable equilibrium relative to $\mathcal{G}$.

(ii) Note that since $x$ is an equilibrium, $x \in \dot{V}^{-1}(0)$. Suppose $T_{x} \mathcal{N}_{x} \cap \mathcal{F}_{x} \subseteq\{0\}$. 
By (iii) of Proposition 6.4, $\mathcal{M}_{x} \subseteq \mathcal{N}_{x}$. Hence $T_{x} \mathcal{M}_{x} \cap \mathcal{F}_{x} \subseteq T_{x} \mathcal{N}_{x} \cap \mathcal{F}_{x} \subseteq\{0\}$. Therefore, by (i), $x$ is a Lyapunov stable equilibrium relative to $\mathcal{G}$.

(iii) Let $\mathcal{U} \subseteq \mathcal{W}$ be a relatively open neighborhood of $x$ such that every equilibrium in $\mathcal{U}$ is a local minimizer of $V$ relative to $\mathcal{K}$ and $T_{z} \mathcal{N}_{z} \cap \mathcal{F}_{z} \subseteq\{0\}$ for every $z \in \mathcal{N} \cap \mathcal{U}$. Since $x$ is a local maximizer of $\dot{V}$ relative to $\mathcal{G}$, we may assume without loss of generality that $\dot{V}(z) \leq \dot{V}(x)=0$ for every $z \in \mathcal{U}$. It follows that every equilibrium in $\mathcal{U}$ is a local maximizer of $\dot{V}$ relative to $\mathcal{G}$. Since every equilibrium in $\mathcal{U}$ is contained in $\mathcal{N}$, it follows from (ii) that every equilibrium in $\mathcal{U}$ is Lyapunov stable relative to $\mathcal{G}$. In particular, $x$ is Lyapunov stable relative to $\mathcal{G}$. By Lyapunov stability of $x$ and local compactness of $\mathcal{G}$, there exists a positively invariant neighborhood $\mathcal{V} \subset \mathcal{U}$ of $x$ that is relatively open and relatively bounded in $\mathcal{G}$, and such that $\overline{\mathcal{V}} \subset \mathcal{U}$. For every $z \in \mathcal{V}, \mathcal{O}_{z} \subseteq \mathcal{V}$ is relatively bounded in $\mathcal{G}$. Therefore, by Propositions 5.1 and 5.3, $\mathcal{O}_{z}^{\infty} \subseteq \overline{\mathcal{V}} \subset \mathcal{W}$ is nonempty and contained in $\overline{\dot{V}^{-1}(0)}$. The invariance of $\mathcal{O}_{z}^{\infty}$ implies that $\mathcal{O}_{z}^{\infty}$ is contained in the largest invariant subset of $\dot{V}^{-1}(0) \cap \mathcal{W}$. Since every invariant set is also negatively invariant, it follows that $\mathcal{O}_{z}^{\infty} \subseteq \mathcal{N}$ for every $z \in \mathcal{V}$. Consider $z \in \mathcal{V}$ and $w \in \mathcal{O}_{z}^{\infty}$. Since $\mathcal{O}_{z}^{\infty}$ is connected and contained in $\mathcal{N}$, it follows that $\mathcal{O}_{z}^{\infty} \subseteq \mathcal{N}_{w}$. Therefore, $T_{w} \mathcal{O}_{z}^{\infty} \cap \mathcal{F}_{w} \subseteq T_{w} \mathcal{N}_{w} \cap \mathcal{F}_{w} \subseteq\{0\}$. Now Proposition 5.2 implies that $\lim _{t \rightarrow \infty} \psi(t, z)$ exists. Since $z \in \mathcal{V}$ was chosen arbitrarily, it follows that every trajectory in $\mathcal{V}$ converges to a limit. The positive invariance of $\mathcal{V}$ implies that the limit of every trajectory in $\mathcal{V}$ is contained in $\overline{\mathcal{V}}$. Since every equilibrium in $\overline{\mathcal{V}} \subset \mathcal{U}$ is Lyapunov stable relative to $\mathcal{G}$, it follows that $x$ is semistable relative to $\mathcal{G}$.

(iv) Suppose $x$ is an isolated equilibrium, and let $\mathcal{U} \subseteq \mathcal{W}$ be a relatively open neighborhood of $x$ such that $T_{z} \mathcal{N}_{z} \cap \mathcal{F}_{z} \subseteq\{0\}$ for every $z \in \mathcal{N} \cap \mathcal{U}$. Without loss of generality, we may assume that $x$ is the only equilibrium in $\mathcal{U}$. Then, it follows from (iii) that $x$ is semistable relative to $\mathcal{G}$. Asymptotic stability now follows by noting that every isolated equilibrium that is semistable relative to $\mathcal{G}$ is asymptotically stable relative to $\mathcal{G}$.

Remark 7.1. Note that Theorem 7.1 does not require $\dot{V}$ to be continuous. However, in the case where $\dot{V}$ is continuous, we have $\overline{\dot{V}^{-1}(0)}=\dot{V}^{-1}(0)$, and the set $\mathcal{K}$ in Theorem 7.1 is the set of points where the Lyapunov derivative is negative. Thus, in the case where $\dot{V}$ is continuous, Theorem 7.1 requires the equilibrium to be a local minimizer of $V$ only relative to the set of points where the Lyapunov function is strictly decreasing along the solutions of (6).

Example 7.1. In this example, we apply Theorem 7.1 to the mass action kinetics (16) of the Michaelis-Menten chemical reaction introduced in Example 5.2.

Recall that the set of equilibrium concentrations of the reaction in the nonnegative orthant $\mathcal{G}$ is $\mathcal{E}=\mathcal{E}_{1} \cup \mathcal{E}_{2}$, where $\mathcal{E}_{1}=\left\{x \in \mathcal{G}: x_{1}=0, x_{2}=0, x_{3}>0\right\}$ and $\mathcal{E}_{2}=\left\{x \in \mathcal{G}: x_{1} \geq 0, x_{2}=0, x_{3}=0\right\}$. Also recall from Example 5.2 that the righthand side $f$ of the differential equation (16) is nontangent to $\mathcal{E}_{1}$ at every point in $\mathcal{E}_{1}$ relative to $\mathcal{G}$.

We claim that every equilibrium in $\mathcal{E}_{1}$ is semistable relative to $\mathcal{G}$. To show this, let $\alpha \in\left(1,1+k_{3} / k_{2}\right)$, and consider the function $V: \mathcal{G} \rightarrow \mathbb{R}$ defined by $V(x)=\alpha x_{1}+x_{2}$. Then, $V(x) \geq 0$ for every $x \in \mathcal{G}$ and $V^{-1}(0)=\overline{\mathcal{E}_{1}}$. Thus every point in $\mathcal{E}_{1}$ is a local minimizer of $V$ relative to $\mathcal{G}$. Since $\dot{V}: \mathcal{G} \rightarrow \mathbb{R}$ is given by $\dot{V}(x)=\left[\alpha k_{2}-\left(k_{2}+k_{3}\right)\right] x_{2}+$ $k_{1}(1-\alpha) x_{1} x_{3}$, it follows that $\dot{V}(x) \leq 0$ for every $x \in \mathcal{G}$ and $\dot{V}^{-1}(0)=\overline{\dot{V}^{-1}(0)}=\mathcal{E}$ so that the largest negatively invariant subset of $\overline{\dot{V}^{-1}(0)}$ is $\mathcal{E}$.

Now consider an equilibrium $x \in \mathcal{E}_{1}$. There exists a relatively open neighborhood $\mathcal{U} \subseteq \mathcal{G}$ of $x$ such that $\mathcal{U} \cap \mathcal{E}=\mathcal{U} \cap \mathcal{E}_{1}$. It now follows that every equilibrium $z \in \mathcal{U} \cap \mathcal{E}$ 
is a local maximizer of $\dot{V}$ and a local minimizer of $V$ relative to $\mathcal{G}$ and, as shown in Example 5.2, that $f$ is nontangent to $\mathcal{E}$ at every $z \in \mathcal{U} \cap \mathcal{E}$ relative to $\mathcal{G}$. Hence, applying (iii) of Theorem 7.1 with $\mathcal{W}=\mathcal{G}$, it follows that every equilibrium in $\mathcal{E}_{1}$ is semistable relative to $\mathcal{G}$.

The following corollary of Theorem 7.1 is an extension of Theorems 1 and 2 from [16].

Corollary 7.1. Suppose $V: \mathcal{G} \rightarrow \mathbb{R}$ is a continuous function such that $\dot{V}$ is defined on $\mathcal{G}$. Let $x \in \mathcal{E}$ be a local maximizer of $\dot{V}$ relative to $\mathcal{G}$ and a local minimizer of $V$ relative to the set $\mathcal{K} \stackrel{\text { def }}{=} \mathcal{G} \backslash \overline{\dot{V}^{-1}(0)}$. Let $\mathcal{W} \subseteq \mathcal{G}$ be a relatively open neighborhood of $x$. For every $z \in \mathcal{W}$, let $\mathcal{M}_{z}$ denote the largest connected subset of $V^{-1}(V(z)) \cap \mathcal{W}$ that is negatively invariant and contains $z$. Let $\mathcal{N}$ denote the largest negatively invariant subset of $\overline{\dot{V}^{-1}(0)} \cap \mathcal{W}$. Then the following statements hold.

(i) If $\mathcal{M}_{x}=\{x\}$, then $x$ is Lyapunov stable relative to $\mathcal{G}$.

(ii) If $x$ is an isolated point of $\mathcal{N}$, then $x$ is asymptotically stable relative to $\mathcal{G}$.

Proof. (i) Suppose $\mathcal{M}_{x}=\{x\}$. Then Lyapunov stability of $x$ follows from (i) of Theorem 7.1 by noting that $T_{x} \mathcal{M}_{x}=\{0\}$.

(ii) Suppose $\{x\}$ is an isolated point of $\mathcal{N}$. Since every equilibrium in $\mathcal{W}$ is contained in $\mathcal{N}$, it follows that $x$ is an isolated equilibrium. Also, the connected component $\mathcal{N}_{x}$ of $\mathcal{N}$ containing $x$ is $\{x\}$, and hence $T_{x} \mathcal{N}_{x}=\{0\}$. The statement (ii) now follows by applying Theorem 7.1, (iv).

Remark 7.2. Theorems 1 and 2 of [16] follow from (i) and (ii) of Corollary 7.1, respectively. However, while Corollary 7.1 requires only that the equilibrium be a local minimizer of the Lyapunov function relative to the set of points at which the Lyapunov function is strictly decreasing, the results of [16] require the equilibrium to be a local minimizer of the Lyapunov function relative to $\mathcal{G}$. Thus Corollary 7.1 is an extension of the main results of [16]. It is shown in [16] that the main result of [1] follows from Theorem 1 in [16]. Since Corollary 7.1 is an extension of the main results of [16], the arguments presented in [16] can be used to show that the main result of [1] follows from Corollary 7.1.

Remark 7.3. It is interesting to note that neither Corollary 7.1 nor the results of [16] can be applied in the case of Example 7.1, because the level sets of $V$ and $\dot{V}$ containing the equilibrium point of interest contain a continuum of equilibria. Consequently, the equilibrium point of interest is not an isolated point of the largest negatively invariant subset of the level sets of $V$ or $\dot{V}$, and the results mentioned above do not apply.

The following corollary of Theorem 7.1 does not require finding negatively invariant subsets of the level sets of the Lyapunov function and its derivative.

Corollary 7.2. Suppose $V: \mathcal{G} \rightarrow \mathbb{R}$ is a continuous function such that $\dot{V}$ is defined on $\mathcal{G}$. Let $x \in \dot{V}^{-1}(0)$ be a local maximizer of $\dot{V}$ relative to $\mathcal{G}$ and a local minimizer of $V$ relative to the set $\mathcal{K} \stackrel{\text { def }}{=} \mathcal{G} \backslash \overline{\dot{V}^{-1}(0)}$. Then the following statements hold.

(i) If $f$ is nontangent to $V^{-1}(V(x))$ at $x$ relative to $\mathcal{G}$, then $x$ is a Lyapunov stable equilibrium relative to $\mathcal{G}$.

(ii) If $f$ is nontangent to $\overline{\dot{V}^{-1}(0)}$ at $x$ relative to $\mathcal{G}$, then $x$ is a Lyapunov stable equilibrium relative to $\mathcal{G}$.

(iii) If there exists a relatively open neighborhood $\mathcal{U} \subseteq \mathcal{G}$ of $x$ such that every equilibrium in $\mathcal{U}$ is a local minimizer of $V$ relative to $\mathcal{K}$ and $f$ is nontangent 
to $\overline{\dot{V}^{-1}(0)}$ at every point in $\mathcal{U} \cap \overline{\dot{V}^{-1}(0)}$ relative to $\mathcal{G}$, then $x$ is a semistable equilibrium relative to $\mathcal{G}$.

(iv) If $x$ is an isolated point of the set $\dot{V}^{-1}(0)$, then $x$ is an asymptotically stable equilibrium relative to $\mathcal{G}$.

Proof. Let $\mathcal{V} \subseteq \mathcal{G}$ be a relatively open neighborhood of $x$ such that $\dot{V}(z) \leq \dot{V}(x)=$ 0 for every $z \in \mathcal{V}$ and $V(x) \leq V(z)$ for every $z \in \mathcal{K} \cap \mathcal{V}$. There exists $\tau>0$ such that $\psi(t, x) \in \mathcal{V}$ for all $t \in[0, \tau)$. Therefore, $V(\psi(t, x)) \geq V(x)$ for every $t \in[0, \tau)$. However, since $\dot{V}(\psi(t, x)) \leq 0$ for every $t \in[0, \tau)$, it follows that $V(\psi(t, x)) \leq V(x)$ for every $t \in[0, \tau)$. We conclude that $V(\psi(t, x))=V(x)$ and $\dot{V}(\psi(t, x))=0$ for every $t \in[0, \tau)$. In other words, $\psi(t, x) \in V^{-1}(V(x)) \cap \dot{V}^{-1}(0)$ for every $t \in[0, \tau)$. In particular, $x \in \dot{V}^{-1}(0)$. Also, if $\left\{t_{i}\right\}$ is a sequence in $[0, \tau)$ that converges to zero, then $\left\{\psi\left(t_{i}, x\right)\right\}$ is a sequence in $V^{-1}(V(x)) \cap \dot{V}^{-1}(0)$ converging to $x$ such that $\lim _{i \rightarrow \infty} \frac{1}{t_{i}}\left[\psi\left(t_{i}, x\right)-x\right]=f(x)$. Thus $f(x) \in T_{x} \dot{V}^{-1}(0) \cap T_{x} V^{-1}(V(x))$.

(i) Suppose $T_{x} V^{-1}(V(x)) \cap \mathcal{F}_{x} \subseteq\{0\}$. If $\mathcal{F}_{x}=\varnothing$, then $x \in \operatorname{int} \mathcal{E}$ and $f(x)=0$. On the other hand, if $\mathcal{F}_{x} \neq \varnothing$, then $f(x) \in T_{x} V^{-1}(V(x)) \cap \mathcal{F}_{x} \subseteq\{0\}$ so that $f(x)=0$. In either case, it follows that $x$ is an equilibrium. Lyapunov stability of $x$ now follows from (i) of Theorem 7.1 by noting that if $\mathcal{M}_{x}$ is the largest connected, negatively invariant subset of $V^{-1}(V(x))$ containing $x$, then $T_{x} \mathcal{M}_{x} \cap \mathcal{F}_{x} \subseteq T_{x} V^{-1}(V(x)) \cap \mathcal{F}_{x} \subseteq$ $\{0\}$.

(ii) Suppose $T_{x} \overline{\dot{V}^{-1}(0)} \cap \mathcal{F}_{x} \subseteq\{0\}$. Arguing as in the proof of (i), it can be shown that $x$ is an equilibrium. Lyapunov stability of $x$ now follows from (ii) of Theorem 7.1 by noting that if $\mathcal{N}_{x}$ is the largest connected, negatively invariant subset of $\overline{\dot{V}^{-1}(0)}$ containing $x$, then $T_{x} \mathcal{N}_{x} \cap \mathcal{F}_{x} \subseteq T_{x} \overline{\dot{V}^{-1}(0)} \cap \mathcal{F}_{x} \subseteq\{0\}$.

(iii) Suppose $\mathcal{U} \subseteq \mathcal{G}$ is a relatively open neighborhood of $x$ such that every equilibrium in $\mathcal{U}$ is a local minimizer of $V$ relative to the set $\mathcal{K}$ and $T_{z} \overline{\dot{V}^{-1}(0)} \cap \mathcal{F}_{z} \subseteq\{0\}$ for every $z \in \mathcal{U} \cap \overline{\dot{V}^{-1}(0)}$. Arguing as in the proof of (i), it can be shown that $x$ is an equilibrium. The result now follows from (iii) of Theorem 7.1 by noting that, if $\mathcal{N}$ is the largest negatively invariant subset of $\overline{\dot{V}^{-1}(0)}$, then, for every $z \in \mathcal{N} \cap \mathcal{U} \subseteq \overline{\dot{V}^{-1}(0)} \cap \mathcal{U}$, it follows that $T_{z} \mathcal{N} \cap \mathcal{F}_{z} \subseteq T_{z} \overline{\dot{V}^{-1}(0)} \cap \mathcal{F}_{z} \subseteq\{0\}$.

(iv) Suppose $x$ is an isolated point of $\dot{V}^{-1}(0)$. Then $x$ is also an isolated point of $\dot{V}^{-1}(0)$. Since $\psi(t, x) \in \dot{V}^{-1}(0)$ for all $t \in[0, \tau)$, it follows from the continuity of $\psi$ that $\psi(t, x)=x$ for all $t \in[0, \tau)$. In other words, $x$ is an equilibrium. Since every equilibrium is contained in $\dot{V}^{-1}(0)$, it follows that $x$ is an isolated equilibrium. Since $x$ is an isolated point of $\overline{\dot{V}^{-1}(0)}$, it follows that $T_{x} \overline{\dot{V}^{-1}(0)}=\{0\}$. Hence (iii) implies that $x$ is semistable relative to $\mathcal{G}$. Since an isolated semistable equilibrium is asymptotically stable, (iv) follows.

Example 7.2. Consider the system described in Example 1.1. Suppose $\alpha \leq \beta$, and let $\mathcal{G}=\mathbb{R}^{2}$. Consider the Lyapunov function $V: \mathcal{G} \rightarrow \mathbb{R}$ given by $V(x)=\frac{1}{4}\left(x_{1}^{2}+\right.$ $\left.x_{2}^{2}-1\right)^{2}$ introduced in Example 5.4, and recall that $\dot{V}(x)=-\left(x_{1}^{2}+x_{2}^{2}\right)\left|x_{1}^{2}+x_{2}^{2}-1\right|^{1+\alpha}$ so that $\dot{V}(x) \leq 0$ for all $x \in \mathcal{G}$ and $\overline{\dot{V}^{-1}(0)}=\mathrm{S}^{1} \cup\{0\}$. The set $\mathcal{K}=\mathcal{G} \backslash \overline{\dot{V}^{-1}(0)}$ in Corollary 7.2 is given in this case by $\mathcal{K}=\mathcal{G} \backslash\left(\mathrm{S}^{1} \cup\{0\}\right)$. Hence every point $x \in \mathrm{S}^{1}$ is a local minimizer of $V$ relative to $\mathcal{K}$. We know from Example 4.2 that $f$ is nontangent to $\mathrm{S}^{1}$ and hence to $\dot{V}^{-1}(0)$ at every $z \in \mathrm{S}^{1}$ relative to $\mathcal{G}$. Hence by (iii) of Corollary 7.2 , it follows that every $x \in \mathrm{S}^{1}$ is semistable relative to $\mathcal{G}=\mathbb{R}^{2}$.

The following theorem gives stability conditions in terms of invariant sets rather than negatively invariant sets as in Theorem 7.1. Also, the first part of the theorem does not require the equilibrium to be a local minimizer of the Lyapunov function. 
TheOREm 7.2. Suppose $V: \mathcal{G} \rightarrow \mathbb{R}$ is a continuous function such that $\dot{V}$ is defined on $\mathcal{G}$. For every $z \in \mathcal{G}$, let $\mathcal{P}_{z}$ denote the largest connected subset of $\{w \in$ $\mathcal{G}: V(w) \leq V(z)\}$ that is invariant and contains $z$. Let $x \in \mathcal{E}$ be a global maximizer of $\dot{V}$ relative to $\mathcal{G}$. Let $\varepsilon>0$, and let $\mathcal{Q}$ be the largest invariant subset of the set $\{w \in \mathcal{G}: V(w)<V(x)+\varepsilon\}$. For every $z \in \mathcal{Q}$, let $\mathcal{Q}_{z}$ denote the connected component of $\mathcal{Q}$ containing $z$. Then the following statements hold.

(i) If $f$ is nontangent to $\mathcal{P}_{x}$ at $x$ relative to $\mathcal{G}$, then $x$ is Lyapunov stable relative to $\mathcal{G}$.

(ii) If there exists a relatively open neighborhood $\mathcal{U} \subseteq \mathcal{G}$ of $x$ such that, for every $z \in \mathcal{U} \cap \mathcal{Q}, f$ is nontangent to $\mathcal{Q}_{z}$ at $z$ relative to $\mathcal{G}$, then $x$ is semistable relative to $\mathcal{G}$.

(iii) If $x$ is an isolated point of $\mathcal{Q}$, then $x$ is asymptotically stable relative to $\mathcal{G}$.

If, in addition, $x$ is a local minimizer of $V$ relative to the set $\mathcal{K} \stackrel{\text { def }}{=} \mathcal{G} \overline{\dot{V}^{-1}(0)}$, then the following statements hold.

(iv) If $f$ is nontangent to $\mathcal{P}_{x} \cap V^{-1}(V(x))$ at $x$ relative to $\mathcal{G}$, then $x$ is Lyapunov stable relative to $\mathcal{G}$.

(v) If $f$ is nontangent to $\mathcal{P}_{x} \cap \overline{\dot{V}^{-1}(0)}$ at $x$ relative to $\mathcal{G}$, then $x$ is Lyapunov stable relative to $\mathcal{G}$.

(vi) If there exists a relatively open neighborhood $\mathcal{U} \subseteq \mathcal{G}$ of $x$ such that every equilibrium in $\mathcal{U}$ is a local minimizer of $V$ relative to the set $\mathcal{K}$ and, for every $z \in \mathcal{U} \cap \mathcal{Q}, f$ is nontangent to $\mathcal{Q}_{z} \cap \overline{\dot{V}^{-1}(0)}$ at $z$ relative to $\mathcal{G}$, then $x$ is semistable relative to $\mathcal{G}$.

Proof. Note that by (i) of Proposition 6.4, $\mathcal{R}_{x} \subseteq \mathcal{P}_{x}$.

(i) Suppose $T_{x} \mathcal{P}_{x} \cap \mathcal{F}_{x} \subseteq\{0\}$. We have $T_{x} \mathcal{R}_{x} \cap \mathcal{F}_{x} \subseteq T_{x} \mathcal{P}_{x} \cap \mathcal{F}_{x} \subseteq\{0\}$. Hence it follows from Proposition 6.3 that $x$ is Lyapunov stable relative to $\mathcal{G}$.

(ii) Let $\mathcal{U} \subseteq \mathcal{G}$ be a relatively open neighborhood of $x$ such that $T_{z} \mathcal{Q}_{z} \cap \mathcal{F}_{z} \subseteq$ $\{0\}$ for every $z \in \mathcal{U} \cap \mathcal{Q}$. Since $V$ is continuous, we can assume without loss of generality that $V(z)<V(x)+\varepsilon$ for every $z \in \mathcal{U}$. Then, for every $z \in \mathcal{U},\{w \in$ $\mathcal{G}: V(w) \leq V(z)\} \subseteq\{w \in \mathcal{G}: V(w)<V(x)+\varepsilon\}$. Consequently, $\mathcal{P}_{z} \subseteq \mathcal{Q}_{z}$ for every $z \in \mathcal{U}$. In particular, if $z \in \mathcal{U}$ is an equilibrium, then $z \in \mathcal{P}_{z} \subseteq \mathcal{Q}_{z}$ so that $T_{z} \mathcal{P}_{z} \cap \mathcal{F}_{z} \subseteq T_{z} \mathcal{Q}_{z} \cap \mathcal{F}_{z} \subseteq\{0\}$. It therefore follows from (i) that every equilibrium in $\mathcal{U}$ is Lyapunov stable relative to $\mathcal{G}$. In particular, $x$ is Lyapunov stable relative to $\mathcal{G}$. By Lyapunov stability of $x$ and local compactness of $\mathcal{G}$, there exists a positively invariant neighborhood $\mathcal{V} \subset \mathcal{U}$ of $x$ that is relatively open and bounded in $\mathcal{G}$ and such that $\overline{\mathcal{V}} \subset \mathcal{U}$. Consider $z \in \mathcal{V}$. Then $\mathcal{O}_{z} \subseteq \mathcal{V}$ is relatively bounded in $\mathcal{G}$, and hence by Propositions 5.1 and 5.3, $\mathcal{O}_{z}^{\infty} \subseteq \overline{\mathcal{V}} \subset \mathcal{U}$ is nonempty, connected, and contained in the largest invariant subset of $\{w \in \mathcal{G}: V(w) \leq V(z)\}$ and hence in $\mathcal{Q}$. Next, let $w \in \mathcal{O}_{z}^{\infty}$. Since $\mathcal{O}_{z}^{\infty} \subseteq \mathcal{Q}$ is connected, it follows that $\mathcal{O}_{z}^{\infty} \subseteq \mathcal{Q}_{w}$. Now, since $w \in \overline{\mathcal{V}} \cap \mathcal{Q} \subseteq \mathcal{U} \cap \mathcal{Q}$, we have $T_{w} \mathcal{O}_{z}^{\infty} \cap \mathcal{F}_{w} \subseteq T_{w} \mathcal{Q}_{w} \cap \mathcal{F}_{w} \subseteq\{0\}$. Proposition 5.2 now implies that $\lim _{t \rightarrow \infty} \psi(t, z)$ exists and is contained in $\overline{\mathcal{V}} \subseteq \mathcal{G}$. Since $z \in \mathcal{V}$ was chosen arbitrarily, it follows that every trajectory in $\mathcal{V}$ converges to a limit. The positive invariance of $\mathcal{V}$ implies that the limit of every trajectory in $\mathcal{V}$ is contained in $\overline{\mathcal{V}}$. Since every equilibrium in $\overline{\mathcal{V}} \subset \mathcal{U}$ is Lyapunov stable relative to $\mathcal{G}$, it follows that $x$ is semistable relative to $\mathcal{G}$.

(iii) Suppose $x$ is an isolated point of $\mathcal{Q}$, and let $\mathcal{U} \subseteq \mathcal{G}$ be a relatively open neighborhood of $x$ such that $\mathcal{U} \cap \mathcal{Q}=\{x\}$. Then $T_{x} \mathcal{Q}=\{0\}$, and hence every point $z \in \mathcal{U} \cap \mathcal{Q}$ satisfies $T_{z} \mathcal{Q} \cap \mathcal{F}_{z} \subseteq\{0\}$. By (ii), $x$ is semistable relative to $\mathcal{G}$. Next, consider the relatively open neighborhood $\mathcal{V}=\{z \in \mathcal{G}: V(z)<V(x)+\varepsilon\}$ of $x$. Since every equilibrium in $\mathcal{V}$ is contained in $\mathcal{Q}$, we have $(\mathcal{U} \cap \mathcal{V}) \cap \mathcal{E}=\mathcal{U} \cap(\mathcal{V} \cap \mathcal{E}) \subseteq \mathcal{U} \cap \mathcal{Q}=\{x\}$. 
Since $\mathcal{U} \cap \mathcal{V}$ is a relatively open neighborhood of $x$, it follows that $x$ is an isolated equilibrium. The result now follows by noting that an isolated equilibrium that is semistable relative to $\mathcal{G}$ is also asymptotically stable relative to $\mathcal{G}$.

Now, suppose $x$ is a local minimizer of $V$ relative to the set $\mathcal{K}=\mathcal{G} \backslash \overline{\dot{V}^{-1}(0)}$. By (ii) and (iii) of Proposition 6.4, there exists a neighborhood $\mathcal{V}$ of $x$ that is open and bounded relative to $\mathcal{G}$, and such that $\mathcal{R}_{x}^{\mathcal{V}} \subseteq V^{-1}(V(x)) \cap \overline{\dot{V}^{-1}(0)}$.

(iv) Suppose $T_{x}\left(\mathcal{P}_{x} \cap V^{-1}(V(x))\right) \cap \mathcal{F}_{x} \subseteq\{0\}$. Then $T_{x} \mathcal{R}_{x}^{\mathcal{V}} \cap \mathcal{F}_{x} \subseteq T_{x}\left(\mathcal{R}_{x} \cap\right.$ $\left.V^{-1}(V(x))\right) \cap \mathcal{F}_{x} \subseteq T_{x}\left(\mathcal{P}_{x} \cap V^{-1}(V(x))\right) \cap \mathcal{F}_{x} \subseteq\{0\}$. It now follows from Proposition 6.3 that $x$ is Lyapunov stable relative to $\mathcal{G}$.

(v) Suppose $T_{x}\left(\mathcal{P}_{x} \cap \overline{\dot{V}^{-1}(0)}\right) \cap \mathcal{F}_{x} \subseteq\{0\}$. Then $T_{x} \mathcal{R}_{x}^{\mathcal{V}} \cap \mathcal{F}_{x} \subseteq T_{x}\left(\mathcal{R}_{x} \cap \overline{\dot{V}^{-1}(0)}\right) \cap$ $\mathcal{F}_{x} \subseteq T_{x}\left(\mathcal{P}_{x} \cap \overline{\dot{V}^{-1}(0)}\right) \cap \mathcal{F}_{x} \subseteq\{0\}$. It now follows from Proposition 6.3 that $x$ is Lyapunov stable relative to $\mathcal{G}$.

(vi) Let $\mathcal{U} \subseteq \mathcal{G}$ be a relatively open neighborhood of $x$ such that every equilibrium in $\mathcal{U}$ is a local minimizer of $V$ relative to the set $\mathcal{K}$, and every point $z$ in $\mathcal{U} \cap \mathcal{Q}$ satisfies $T_{z}\left(\mathcal{Q}_{z} \cap \overline{\dot{V}^{-1}(0)}\right) \cap \mathcal{F}_{z} \subseteq\{0\}$. Without loss of generality, we assume that $V(z)<V(x)+\varepsilon$ for every $z \in \mathcal{U}$. Then, for every $z \in \mathcal{U}, \mathcal{P}_{z} \subseteq \mathcal{Q}_{z}$ and hence $T_{z}\left(\mathcal{P}_{z} \cap\right.$ $\left.\overline{\dot{V}^{-1}(0)}\right) \cap \mathcal{F}_{z} \subseteq T_{z}\left(\mathcal{Q}_{z} \cap \overline{\dot{V}^{-1}(0)}\right) \cap \mathcal{F}_{z} \subseteq\{0\}$. It therefore follows from (v) that every equilibrium in $\mathcal{U}$ is Lyapunov stable relative to $\mathcal{G}$. In particular, $x$ is Lyapunov stable relative to $\mathcal{G}$. By Lyapunov stability of $x$ and local compactness of $\mathcal{G}$, there exists a positively invariant, relatively open, and relatively bounded neighborhood $\mathcal{V} \subset \mathcal{G}$ of $x$ such that $\overline{\mathcal{V}} \subset \mathcal{U}$. Consider $z \in \mathcal{V}$. Then $\mathcal{O}_{z} \subseteq \mathcal{V}$ is relatively bounded in $\mathcal{G}$, and hence by Propositions 5.1 and $5.3, \mathcal{O}_{z}^{\infty} \subseteq \overline{\mathcal{V}}$ is nonempty, connected, and contained in the largest invariant subset of $\{w \in \mathcal{G}: V(w) \leq V(z)\} \subset\{w \in \mathcal{G}: V(w)<V(x)+\epsilon\}$ and hence in $\mathcal{Q}$. Next, let $w \in \mathcal{O}_{z}^{\infty}$. Since $\mathcal{O}_{z}^{\infty} \subseteq \mathcal{Q}$ is connected, it follows that $\mathcal{O}_{z}^{\infty} \subseteq \mathcal{Q}_{w}$. By Proposition 5.3, $\mathcal{O}_{z}^{\infty}$ is also contained in $\dot{V}^{-1}(0)$. Now, since $w \in \overline{\mathcal{V}} \cap \mathcal{Q} \subseteq \mathcal{U} \cap \mathcal{Q}$, we have $T_{w} \mathcal{O}_{z}^{\infty} \cap \mathcal{F}_{w} \subseteq T_{w}\left(\mathcal{Q}_{w} \cap \dot{V}^{-1}(0)\right) \cap \mathcal{F}_{w} \subseteq T_{w}\left(\mathcal{Q}_{w} \cap \overline{\dot{V}^{-1}(0)}\right) \cap \mathcal{F}_{w} \subseteq\{0\}$. Proposition 5.2 now implies that $\lim _{t \rightarrow \infty} \psi(t, z)$ exists and is contained in $\mathcal{G}$. Since $z \in \mathcal{V}$ was chosen to be arbitrary, it follows that every trajectory in $\mathcal{V}$ converges to an equilibrium in $\overline{\mathcal{V}} \subset \mathcal{U}$. Semistability of $x$ relative to $\mathcal{G}$ now follows by noting that every equilibrium in $\overline{\mathcal{V}} \subset \mathcal{U}$ is Lyapunov stable relative to $\mathcal{G}$.

Example 7.3. In this example, we use Theorem 7.2 to show that every equilibrium $(0, k)$ of the adaptive closed-loop system (10)-(11) satisfying $g^{\prime}(k)=a+b k^{2} \cos k>0$ is semistable, where $g^{\prime}$ is the derivative of the function $g: k \mapsto a k+b\left(k^{2}-2\right) \sin k+$ $2 b k \cos k$ introduced in Example 3.1.

Suppose $k_{0} \in \mathbb{R}$ is such that $g^{\prime}\left(k_{0}\right)>0$. Since $g^{\prime}$ is continuous, there exist $\delta>0$ and $r>0$ such that $g^{\prime}(k)>r$ for all $k \in\left[k_{0}-\delta, k_{0}+\delta\right]$. In particular, we note that $g$ is increasing on the interval $\left[k_{0}-\delta, k_{0}+\delta\right]$.

We claim that the equilibrium $\left(0, k_{0}\right)$ of the system $(10)-(11)$ is semistable. To show this, consider the Lyapunov function $V(y, k)=e^{-k}$ introduced in Example 5.3, and let $\varepsilon=V\left(0, k_{0}\right)\left(e^{\delta}-1\right)$. It is easy to see that the set $\left\{(y, k) \in \mathbb{R}^{2}: V(y, k) \leq\right.$ $\left.V\left(0, k_{0}\right)+\varepsilon\right\}=\left\{(y, k) \in \mathbb{R}^{2}: k \geq k_{0}-\delta\right\}$. Let $\mathcal{Q}$ denote the largest invariant subset of $\left\{(y, k) \in \mathbb{R}^{2}: k \geq k_{0}-\delta\right\}$. Also, recall from Example 5.3 that $\dot{V}(y, k)=-e^{-k} y^{2}$ and $\dot{V}^{-1}(0)=\{(0, k): k \in \mathbb{R}\}=\mathcal{E}$.

Let $U: \mathbb{R}^{2} \rightarrow \mathbb{R}$ be the function $U(y, k)=\frac{1}{2} y^{2}+g(k)$, and recall from Example 3.1 that $U$ is weakly proper and $\dot{U} \equiv 0$. Choose $k_{1} \in\left(k_{0}, k_{0}+\delta\right)$, and let $c=U\left(0, k_{1}\right)=$ $g\left(k_{1}\right)$. Note that since $g$ is increasing on $\left[k_{0}, k_{0}+\delta\right], U\left(0, k_{0}\right)=g\left(k_{0}\right)<g\left(k_{1}\right)=c$. Let $\mathcal{U}$ be the connected component of the set $\left\{(y, k) \in \mathbb{R}^{2}: U(y, k)<c\right\}$ containing $\left(0, k_{0}\right)$. The set $\mathcal{U}$ is open by continuity and bounded by weak properness of $U$. 
Moreover, letting $\rho$ denote the projection $(y, k) \mapsto k$, the set $\rho(\mathcal{U})$ is a connected set that contains $k_{0}$ and does not contain $k_{1}>k_{0}$. Hence we conclude that, for every $(y, k) \in \mathcal{U}, k<k_{1}<k_{0}+\delta$. It follows that every $(y, k) \in \mathcal{U} \cap \mathcal{Q}$ satisfies $k \in\left[k_{0}-\delta, k_{0}+\delta\right]$. Also, since $\mathcal{U}$ is bounded, it follows that there exists $Y \geq 0$ such that every $(y, k) \in \mathcal{U}$ satisfies $|y| \leq Y$.

We claim that $\mathcal{U} \cap \mathcal{Q}$ is contained in $\dot{V}^{-1}(0)$. To see this, consider $(y, k) \in$ $\mathcal{U} \cap \mathcal{Q}$, and let $\tau>0$. By the invariance of $\mathcal{Q}$, there exists $\left(y_{2}, k_{2}\right) \in \mathcal{Q}$ such that $\left(\phi_{1}(\tau), \phi_{2}(\tau)\right)=(y, k)$, where $\phi: \mathbb{R} \rightarrow \mathbb{R}^{2}$ denotes the solution of (10)-(11) satisfying $\phi(0)=\left(y_{2}, k_{2}\right)$. Since $\dot{U} \equiv 0, U(\phi(t))=U(y, k)<c$ for every $t \in[0, \tau]$. Since, for every $t \in[0, \tau]$, both $(y, k)$ and $\phi(t)$ are contained in the connected subset $\phi([0, \tau])$ of the set $\{(y, k): U(y, k)<c\}$, it follows that $\phi(t) \in \mathcal{U}$ for every $t \in \mathcal{U}$. Thus $\phi(t) \in \mathcal{U} \cap \mathcal{Q}$ for every $t \in[0, \tau]$. Consequently, $\phi_{2}(t) \in\left[k_{0}-\delta, k_{0}+\delta\right]$ for all $t \in[0, \tau]$. Next we compute $\frac{d}{d t}\left(\phi_{1}(t)\right)^{2}=-2 g^{\prime}\left(\phi_{2}(t)\right)\left(\phi_{1}(t)\right)^{2}$ so that $y^{2}=\left(\phi_{2}(\tau)\right)^{2}=$ $y_{2}^{2} \exp \left[-2 \int_{0}^{\tau} g^{\prime}\left(\phi_{2}(t)\right) d t\right] \leq Y^{2} \exp (-2 r \tau)$. Since $\tau>0$ was chosen arbitrarily, it follows that $y=0$. Thus $\mathcal{U} \cap \mathcal{Q} \subseteq \dot{V}^{-1}(0)$.

It was shown in Example 5.3 that, for every $(y, k) \in \dot{V}^{-1}(0)$ such that $g^{\prime}(k) \neq 0$, the vector field $f$ defined by the right-hand side of (10)-(11) is nontangent to $\dot{V}^{-1}(0)$. Hence, for every point $(0, k) \in \mathcal{U} \cap \mathcal{Q}, T_{z} \mathcal{Q} \cap \mathcal{F}_{z}=T_{z}(\mathcal{U} \cap \mathcal{Q}) \cap \mathcal{F}_{z} \subseteq T_{z}\left(\dot{V}^{-1}(0)\right) \cap \mathcal{F}_{z} \subseteq$ $\{0\}$. Thus $f$ is nontangent to $\mathcal{Q}$ at every point in $\mathcal{U} \cap \mathcal{Q}$. By (ii) of Theorem 7.2, we conclude that the equilibrium $\left(0, k_{0}\right)$ is semistable.

Remark 7.4. It is worthwhile to note that Theorem 7.1 cannot be applied in Example 7.3 using the Lyapunov function $V$, because none of the equilibria of the closed-loop system (10)-(11) are local minimizers of the function $V$. In fact, the Lyapunov function $V$ chosen in the example does not have any local minimizers.

Remark 7.5. The convergence of the closed-loop system (10)-(11) is also proved in [17, p. 46]. However, unlike the proof given in [17], our proof of convergence given in Example 5.3 is based on Lyapunov analysis. Moreover, in Example 7.3 we go beyond convergence and identify a class of semistable equilibria for the closed-loop system.

Remark 7.6. Theorems 7.1 and 7.2 involve hypotheses on the Lyapunov function and its derivative that are weaker than sign definiteness or sign semidefiniteness. For instance, in Theorem 7.1 and (iv)-(vi) of Theorem 7.2, the Lyapunov function is required to have a local nonstrict minimum at the equilibrium point only with respect to the set of points where the Lyapunov function derivative is negative, while in (i)-(iii) of Theorem 7.2, the Lyapunov function is not required to have even a local minimum at the equilibrium point of interest. Consequently, Theorems 7.1 and 7.2 can be used to deduce stability of all equilibria in a continuum by considering a single Lyapunov function for all the equilibria. This makes Theorems 7.1 and 7.2 particularly suited for applications to systems having a continuum of equilibria. Examples 7.1 and 7.3 illustrate these observations.

Our final result is the following corollary of Theorem 7.2.

Corollary 7.3. Suppose $V: \mathcal{G} \rightarrow \mathbb{R}$ is a continuous function such that $\dot{V}$ is defined on $\mathcal{G}$. Let $x \in \mathcal{E}$ be a global minimizer of $V$ relative to $\mathcal{G}$ and a global maximizer of $\dot{V}$ relative to $\mathcal{G}$. Let $\mathcal{P}$ denote the largest subset of $V^{-1}(V(x))$ that is invariant and connected and contains $x$. Let $\mathcal{N}$ denote the largest invariant subset of $\dot{V}^{-1}(0)$. Then the following statements hold.

(i) If $\mathcal{P}=\{x\}$, then $x$ is Lyapunov stable relative to $\mathcal{G}$.

(ii) If $x$ is an isolated point of $\mathcal{N}$, then $x$ is asymptotically stable relative to $\mathcal{G}$. 
Proof. (i) Since $x$ is a global minimizer of $V$ relative to $\mathcal{G}$, it follows that $\mathcal{P}$ is also the largest connected subset of the set $\{z \in \mathcal{G}: V(z) \leq V(x)\}$ that is invariant and contains $x$. If $\mathcal{P}=\{x\}$, then $T_{x} \mathcal{P} \cap \mathcal{F}_{x} \subseteq\{0\}$, and hence the result follows from (i) of Theorem 7.2.

(ii) Since $\mathcal{P}$ is an invariant subset of a level set of $V$, it follows that $\mathcal{P} \subseteq \dot{V}^{-1}(0)$. Thus $\mathcal{P}$ is a connected invariant subset of $\bar{V}^{-1}(0)$ containing $x$, and hence $\mathcal{P} \subseteq \mathcal{N}$. Now suppose $x$ is an isolated point of $\mathcal{N}$. Then $\mathcal{P}=\{x\}$, and hence, by (i), $x$ is Lyapunov stable relative to $\mathcal{G}$. By Lyapunov stability of $x$ and local compactness of $\mathcal{G}$, there exists a relatively open, relatively bounded, and positively invariant neighborhood $\mathcal{U} \subseteq \mathcal{G}$ of $x$ such that $\overline{\mathcal{U}} \cap \mathcal{N}=\{x\}$. For every $z \in \mathcal{U}, \mathcal{O}_{z} \subseteq \mathcal{U}$ is relatively bounded. By Proposition 5.3, for every $z \in \mathcal{U}, \mathcal{O}_{z}^{\infty} \subseteq \overline{\mathcal{O}_{z}} \subseteq \overline{\mathcal{U}}$ is contained in $\mathcal{N}$. Since $\overline{\mathcal{U}} \cap \mathcal{N}=\{x\}$, it follows that $\mathcal{O}_{z}^{\infty}=\{x\}$ for every $z \in \mathcal{U}$. Asymptotic stability of $x$ now follows.

Remark 7.7. It is interesting to compare (ii) of Corollary 7.3 with the KrasovskiiLaSalle theorem for asymptotic stability [20, Thm. 14.1]. In the result from [20], the Lyapunov function and its derivative are assumed to be locally positive definite and locally negative semidefinite, respectively, at the equilibrium. In other words, the equilibrium is assumed to be a strict local minimizer for the Lyapunov function and a local nonstrict maximizer for the Lyapunov function derivative. On the other hand, Corollary 7.3 assumes the equilibrium to be a global nonstrict minimizer and a global nonstrict maximizer for the Lyapunov function and its derivative, respectively. In other words, Corollary 7.3 assumes the Lyapunov function to be only positive semidefinite globally instead of positive definite locally at the equilibrium. Thus, both (ii) of Corollary 7.3 and Theorem 14.1 of [20] assert asymptotic stability of the equilibrium under two alternative and complementary sets of assumptions.

Remark 7.8. It is also interesting to compare Corollary 7.3 with Corollary 7.1. While Corollary 7.3 requires the equilibrium to be a global minimizer and a global maximizer of $V$ and $\dot{V}$, respectively, Corollary 7.1 requires only that the equilibrium be a local minimizer and a local maximizer, respectively, of $V$ and $\dot{V}$. Thus the hypotheses of Corollary 7.1 on the Lyapunov function and its derivative are weaker than those of Corollary 7.3. However, Corollary 7.1 requires the equilibrium point to be an isolated point of the largest negatively invariant subsets of level sets of $V$ and $\dot{V}$, while Corollary 7.3 requires only that the equilibrium be an isolated point of the largest invariant subsets of level sets of $V$ and $\dot{V}$. Since invariant sets are also negatively invariant, it is clear that the hypotheses of Corollary 7.3 relating to invariant subsets are weaker than the corresponding hypotheses in Corollary 7.1. Thus both corollaries assert Lyapunov and asymptotic stability under alternative and complementary sets of conditions.

8. Conclusions. This paper introduces convergence and semistability as two notions of importance in the study of systems having a continuum of equilibria. The main contribution of this paper has been the introduction of the notion of nontangency and its application in the Lyapunov analysis of systems having a continuum of equilibria. Positive limit sets and restricted prolongations play a key role in the application of nontangency to Lyapunov analysis of convergence and stability, respectively. We introduce restricted prolongations in the paper, establish their invariance properties, and give inclusion results for restricted prolongations in terms of invariant and negatively invariant subsets of the level and sublevel sets of a Lyapunov function and its derivative. Using nontangency, we obtain Lyapunov results for convergence, Lyapunov stability, semistability, and asymptotic stability. The results on Lyapunov 
stability and asymptotic stability involve hypotheses on the Lyapunov function and its derivative that are weaker than sign definiteness or semidefiniteness. This makes our results particularly suited for applications to systems having a continuum of equilibria. The weaker hypotheses on the Lyapunov function and its derivative are supplemented by assuming nontangency of the vector field to appropriate subsets of the level and sublevel sets of the Lyapunov function and its derivative. We illustrate the main results by applying them to an example from chemical kinetics and an example from adaptive control.

Appendix. Proof of Proposition 4.2. We present here the proof of Proposition 4.2. First, we recall some definitions related to set convergence $[2,26]$. Consider a sequence $\left\{\mathcal{W}_{k}\right\}$ of subsets of $\mathbb{R}^{n}$. The limit superior of the sequence, denoted $\lim \sup _{k \rightarrow \infty} \mathcal{W}_{k}$, is the set of all subsequential limits of sequences $\left\{w_{k}\right\}$ in $\mathbb{R}^{n}$ such that $w_{k} \in \mathcal{W}_{k}$ for every $k$. The limit inferior of the sequence, denoted by $\liminf \operatorname{in}_{k \rightarrow \infty} \mathcal{W}_{k}$, is the set of limits of convergent sequences $\left\{w_{k}\right\}$ in $\mathbb{R}^{n}$ such that $w_{k} \in \mathcal{W}_{k}$ for every $k$. The sequence $\left\{\mathcal{W}_{k}\right\}$ converges to the set $\mathcal{W} \subseteq \mathbb{R}^{n}$ if $\mathcal{W}=\liminf _{k \rightarrow \infty} \mathcal{W}_{k}=\limsup _{k \rightarrow \infty} \mathcal{W}_{k}$.

The proof of Proposition 4.2 requires two basic results on set convergence. The first result is that if $\left\{\mathcal{W}_{k}\right\}$ is a sequence of subsets of a bounded subset of $\mathbb{R}^{n}$, then the sequence $\left\{\mathcal{W}_{k}\right\}$ has a subsequence that converges to a nonempty set. This result follows from Theorem 1.1.7 of [2] and Theorem 4.18 of [26]. The second result is given by the following lemma.

Lemma A.1. Suppose $\left\{\mathcal{W}_{k}\right\}$ is a sequence of connected subsets of a bounded set $\mathcal{B} \subset \mathbb{R}^{n}$ that converges to a set $\mathcal{W} \subseteq \mathbb{R}^{n}$. Then $\mathcal{W}$ is connected.

Proof. Suppose $\mathcal{W}$ is not connected. Then there exist two disjoint open sets $\mathcal{U} \subseteq \mathbb{R}^{n}$ and $\mathcal{V} \subseteq \mathbb{R}^{n}$ such that $\mathcal{W} \subseteq \mathcal{U} \cup \mathcal{V}$ and $\mathcal{W} \cap \mathcal{U}$ and $\mathcal{W} \cap \mathcal{V}$ are nonempty. Since $\mathcal{W}=\liminf _{k \rightarrow \infty} \mathcal{W}_{k}$, there exist convergent sequences $\left\{u_{k}\right\}$ and $\left\{v_{k}\right\}$ in $\mathbb{R}^{n}$ such that $\lim _{k \rightarrow \infty} u_{k} \in \mathcal{W} \cap \mathcal{U}, \lim _{k \rightarrow \infty} v_{k} \in \mathcal{W} \cap \mathcal{V}$ and, for every $k, u_{k}, v_{k} \in \mathcal{W}_{k}$. Therefore, there exists $K>0$ such that, for every $k>K, \mathcal{W}_{k} \cap \mathcal{U}$ and $\mathcal{W}_{k} \cap \mathcal{V}$ are nonempty. Since each $\mathcal{W}_{k}$ is connected, it follows that, for every $k>K$, there exists $w_{k} \in \mathcal{W}_{k}$ such that $w_{k} \notin \mathcal{U} \cup \mathcal{V}$. The sequence $\left\{w_{k}\right\}_{k=K}^{\infty}$ is contained in $\mathcal{B}$ and hence bounded. Let $w$ be a subsequential limit of the sequence $\left\{w_{k}\right\}_{k=K}^{\infty}$. Since the sequence $\left\{w_{k}\right\}_{k=K}^{\infty}$ is contained in the closed set $\mathbb{R}^{n} \backslash(\mathcal{U} \cup \mathcal{V})$, it follows that $w \in \mathbb{R}^{n} \backslash(\mathcal{U} \cup \mathcal{V})$. On the other hand, $w \in \lim \sup _{k \rightarrow \infty} \mathcal{W}_{k}=\mathcal{W} \subseteq \mathcal{U} \cup \mathcal{V}$, which leads to a contradiction. Hence we conclude that $\mathcal{W}$ is connected.

Proof of Proposition 4.2. Suppose $0 \notin \operatorname{co} \mathcal{L}_{x}$. For each $k=1,2, \ldots$, let $\mathcal{U}_{k}=$ $\left\{w \in \mathbb{R}^{n}: \operatorname{dist}\left(w, \mathcal{L}_{x}\right)<1 / k\right\}$. For every $k, \mathcal{U}_{k}$ is an open set containing $\mathcal{L}_{x}, \overline{\mathcal{U}_{k}}$ is compact, and $\overline{\mathcal{U}_{k+1}} \subset \mathcal{U}_{k}$. Moreover, $\cap_{k} \overline{\mathcal{U}_{k}}=\mathcal{L}_{x}$.

For every $k$, there exists a relatively open neighborhood $\mathcal{V}_{k} \subseteq \mathcal{G}$ of $x$ such that, for every $z \in \mathcal{V}_{k} \backslash \mathcal{E},\|f(z)\|^{-1} f(z) \in \mathcal{U}_{k}$. It is easy to show that, for every $k$, every connected component of $f\left(\mathcal{V}_{k}\right) \backslash\{0\}$ is contained in the cone generated by a connected component of $\mathcal{U}_{k}$. Hence it follows that, for every $k, \overline{\operatorname{coco}\left(f\left(\mathcal{V}_{k}\right) \backslash\{0\}\right)} \subseteq \overline{\operatorname{coco} \mathcal{U}_{k}}$. Consequently, $\mathcal{F}_{x} \subseteq \cap_{k=1}^{\infty} \overline{\operatorname{coco}\left(f\left(\mathcal{V}_{k}\right) \backslash\{0\}\right)} \subseteq \cap_{k=1}^{\infty} \overline{\operatorname{coco} \mathcal{U}_{k}}$.

We claim that $\cap_{k=1}^{\infty} \operatorname{coco} \mathcal{U}_{k} \subseteq$ coco $\mathcal{L}_{x}$. To prove this, choose $v \in \cap_{k=1}^{\infty} \overline{\operatorname{coco} \mathcal{U}_{k}}$. There exist a sequence $\left\{\alpha_{k}\right\}$ in $(0, \infty)$ and a sequence $\left\{v_{k}\right\}$ in $\mathbb{R}^{n}$ such that, for every $k, v_{k} \in \operatorname{co} \mathcal{U}_{k}$ and

$$
\left\|v-\alpha_{k} v_{k}\right\|<\frac{1}{k}
$$

For each $k$, let $\mathcal{W}_{k}$ be a connected component of $\mathcal{U}_{k}$ such that $v_{k}$ is contained in the convex hull of $\mathcal{W}_{k}$. Each $\mathcal{W}_{k}$ is a subset of the bounded set $\mathcal{U}_{1}$. Hence there 
exists an increasing sequence $\left\{k_{j}\right\}_{j=1}^{\infty}$ of integers such that the subsequence $\left\{\mathcal{W}_{k_{j}}\right\}_{j=1}^{\infty}$ converges. Let $\mathcal{W}=\lim _{j \rightarrow \infty} \mathcal{W}_{k_{j}}$. Then, by Lemma A.1, $\mathcal{W}$ is connected.

Next, consider $w \in \mathcal{W}$. There exists a sequence $\left\{w_{j}\right\}$ such that $w_{j} \in \mathcal{W}_{k_{j}} \subseteq \mathcal{U}_{k_{j}}$ for every $j$, and $\lim _{j \rightarrow \infty} w_{j}=w$. Since $\left\{\mathcal{U}_{k}\right\}$ is a decreasing sequence of sets, for every $k$, the sequence $\left\{w_{j}\right\}$ is eventually contained in $\mathcal{U}_{k}$. Hence $w \in \overline{\mathcal{U}_{k}}$ for every $k$; that is, $w \in \cap_{k} \overline{\mathcal{U}_{k}}=\mathcal{L}_{x}$. Since $w \in \mathcal{W}$ was arbitrary, it follows that $\mathcal{W} \subseteq \mathcal{L}_{x}$. Hence the connected set $\mathcal{W}$ is contained in a connected component of $\mathcal{L}_{x}$.

By Caratheodory's theorem [25, Thm. 17.1], for every $j$, there exist vectors $w_{j}^{i} \in \mathcal{W}_{k_{j}}, i=1, \ldots, n$, and scalars $\lambda_{j}^{i} \in[0,1], i=1, \ldots, n$, such that $\lambda_{j}^{1}+\cdots+\lambda_{j}^{n}=1$ and

$$
v_{k_{j}}=\lambda_{j}^{1} w_{j}^{1}+\cdots+\lambda_{j}^{n} w_{j}^{n} .
$$

For each $i=1, \ldots, n$, let $\lambda^{i} \in[0,1]$ and $w^{i}$ be subsequential limits of the bounded sequences $\left\{\lambda_{j}^{i}\right\}_{j=1}^{\infty}$ and $\left\{w_{j}^{i}\right\}_{j=1}^{\infty}$, respectively. Then, for every $i, w^{i} \in \mathcal{W}$, while $\lambda^{1}+\cdots+\lambda^{n}=1$. Let $w=\lambda^{1} w^{1}+\cdots+\lambda^{n} w^{n}$. Then $w \in \operatorname{co} \mathcal{L}_{x}$, and hence $w \neq 0$.

By (18), there exists an increasing sequence $\left\{i_{m}\right\}_{m=1}^{\infty}$ of integers such that, for every $m$,

$$
\left\|v_{i_{m}}-w\right\|<\frac{1}{m}
$$

For every $m$, (17) implies that $\alpha_{i_{m}}\left\|v_{i_{m}}\right\| \leq\|v\|+i_{m}^{-1}$, while (19) implies that $\left\|v_{i_{m}}\right\| \geq$ $\|w\|-m^{-1}$. It therefore follows that, for every $m>\|w\|^{-1}, \alpha_{i_{m}} \leq\left(\|v\|+i_{m}^{-1}\right)(\|w\|-$ $\left.m^{-1}\right)^{-1}$. There exists $M>0$ such that, for every $m>M, i_{m}^{-1}<\|v\|$ and $m^{-1}<$ $\|w\| / 2$. Then, for every $m>M, \alpha_{i_{m}} \leq 4\|v\| /\|w\|$. It follows that the subsequence $\left\{\alpha_{i_{m}}\right\}_{m=1}^{\infty}$ is bounded. Hence, by choosing subsequences appropriately, we may assume that there exists $\alpha \in[0, \infty)$ such that, for every $m>M,\left|\alpha_{i_{m}}-\alpha\right|<m^{-1}$. Then, for every $m>M,\|v-\alpha w\| \leq\left\|v-\alpha_{i_{m}} v_{i_{m}}\right\|+\alpha_{i_{m}}\left\|v_{i_{m}}-w\right\|+\left|\alpha_{i_{m}}-\alpha\right|\|w\| \leq$ $i_{m}^{-1}+m^{-1}\left(\alpha_{i_{m}}+\|w\|\right)$. Since the subsequence $\left\{\alpha_{i_{m}}\right\}_{m=1}^{\infty}$ is bounded and the sequence $\left\{i_{m}\right\}$ is divergent, $m$ can be chosen to make the right-hand side of the previous inequality arbitrarily small. It follows that $v=\alpha w$ and thus $v \in \operatorname{coco} \mathcal{L}_{x}$. The result now follows.

\section{REFERENCES}

[1] D. Aeyels and R. Sepulchre, Stability for dynamical systems with first integrals: A topological criterion, Systems Control Lett., 19 (1992), pp. 461-465.

[2] J.-P. Aubin and H. Frankowska, Set-Valued Analysis, Birkhäuser Boston, Boston, 1990.

[3] D. S. Bernstein and S. P. Bhat, Lyapunov stability, semistability, and asymptotic stability of matrix second-order systems, ASME Trans. J. Vibr. Acoustics, 117 (1995), pp. 145-153.

[4] D. S. Bernstein And S. P. Bhat, Nonnegativity, reducibility and semistability of mass action kinetics, in Proceedings of the IEEE Conference on Decision and Control (Phoenix, AZ), IEEE Control Systems Society, Piscataway, NJ, 1999, pp. 2206-2211.

[5] S. P. Bhat And D. S. Bernstein, Lyapunov analysis of semistability, in Proceedings of the American Control Conference, San Diego, CA, 1999, pp. 1608-1612.

[6] S. P. Bhat And D. S. Bernstein, Nontangency-based Lyapunov tests for stability and convergence, in Proceedings of the American Control Conference, Arlington, VA, 2001, pp. 4840-4845.

[7] N. P. Bhatia and O. Hajek, Local Semi-Dynamical Systems, Springer-Verlag, Berlin, 1969.

[8] N. P. Bhatia And G. P. Szegö, Stability Theory of Dynamical Systems, Springer-Verlag, Berlin, 1970.

[9] S. L. Campbell and N. J. Rose, Singular perturbation of autonomous linear systems, SIAM J. Math. Anal., 10 (1979), pp. 542-551. 
[10] J. Dugundu, Topology, Wm. C. Brown, Dubuque, Iowa, 1989.

[11] P. Erdi And J. Toth, Mathematical Models of Chemical Reactions: Theory and Applications of Deterministic and Stochastic Models, Princeton University Press, Princeton, NJ, 1988.

[12] M. Feinberg, The existence and uniqueness of steady states for a class of chemical reaction networks, Arch. Ration. Mech. Anal., 132 (1995), pp. 311-370.

[13] V. Guillemin and A. Pollack, Differential Topology, Prentice-Hall, Englewood Cliffs, NJ, 1974.

[14] P. Hartman, Ordinary Differential Equations, 2nd ed., Birkhäuser Boston, Cambridge, MA, 1982.

[15] U. Helmcke and J. B. Moore, Optimization and Dynamical Systems, Springer-Verlag, London, 1994.

[16] A. IgGidr, B. Kalitine, And R. Outbib, Semidefinite Lyapunov functions stability and stabilization, Math. Control Signals Systems, 9 (1996), pp. 95-106.

[17] A. Ilchmann, Non-Indentifier-Based High Gain Adaptive Control, Springer-Verlag, London, 1993.

[18] J. A. Jacquez And C. P. Simon, Qualitative theory of compartmental systems, SIAM Rev., 35 (1993), pp. 43-79.

[19] H. K. KhaliL, Nonlinear Systems, 2nd ed., Prentice-Hall, Upper Saddle River, NJ, 1996.

[20] N. N. KrasovskiI, Problems of the Theory of Stability of Motion, Stanford University Press, Stanford, CA, 1963

[21] J. P. LaSalle, Some extensions of Liapunov's second method, IRE Trans., CT-7 (1960), pp. $520-527$.

[22] D. R. Mudgett And A. S. Morse, Adaptive stabilization of linear systems with unknown high-frequency gain, IEEE Trans. Automat. Control, 30 (1985), pp. 549-554.

[23] K. S. Narendra And A. M. Annaswamy, Stable Adaptive Systems, Prentice-Hall, Englewood Cliffs, NJ, 1989.

[24] R. D. Nussbaum, Some remarks on a conjecture in parameter adaptive control, Systems Control Lett., 3 (1983), pp. 243-246.

[25] R. T. Rockafellar, Convex Analysis, Princeton University Press, Princeton, NJ, 1970.

[26] R. T. Rockafellar and R. J.-B. Wets, Variational Analysis, Springer-Verlag, Berlin, 1998.

[27] S. K. Sсотт, Chemical Chaos, Oxford University Press, Oxford, UK, 1991.

[28] E. D. Sontag, Structure and stability of certain chemical networks and applications to the kinetic proofreading model of T-cell receptor signal transduction, IEEE Trans. Automat. Control, 46 (2001), pp. 1028-1047.

[29] J. WARGA, Optimal Control of Differential and Functional Equations, Academic Press, New York, 1972.

[30] T. Yoshizawa, Stability Theory and the Existence of Periodic Solutions and Almost Periodic Solutions, Springer-Verlag, New York, 1975. 